\title{
On the Fine Spectrum of the Operator Defined by the Lambda Matrix over the Spaces of Null and Convergent Sequences
}

\author{
Medine Yeşilkayagil ${ }^{1}$ and Feyzi Başar ${ }^{2}$ \\ ${ }^{1}$ Department of Mathematics, Uşak University, 1 Eylül Campus, 64200 Uşak, Turkey \\ ${ }^{2}$ Department of Mathematics, Fatih University, The Hadimköy Campus, Büyükçekmece, 34500 Istanbul, Turkey
}

Correspondence should be addressed to Feyzi Başar; feyzibasar@gmail.com

Received 28 September 2012; Accepted 18 January 2013

Academic Editor: Pavel Kurasov

Copyright @ 2013 M. Yeşilkayagil and F. Başar. This is an open access article distributed under the Creative Commons Attribution License, which permits unrestricted use, distribution, and reproduction in any medium, provided the original work is properly cited.

The main purpose of this paper is to determine the fine spectrum with respect to Goldberg's classification of the operator defined by the lambda matrix over the sequence spaces $c_{0}$ and $c$. As a new development, we give the approximate point spectrum, defect spectrum, and compression spectrum of the matrix operator $\Lambda$ on the sequence spaces $c_{0}$ and $c$. Finally, we present a Mercerian theorem. Since the matrix $\Lambda$ is reduced to a regular matrix depending on the choice of the sequence $\left(\lambda_{k}\right)$ having certain properties and its spectrum is firstly investigated, our work is new and the results are comprehensive.

\section{Introduction}

Let $X$ and $Y$ be Banach spaces, and let $T: X \rightarrow Y$ also be a bounded linear operator. By $R(T)$, we denote the range of $T$; that is,

$$
R(T)=\{y \in Y: y=T x, x \in X\} .
$$

By $B(X)$, we also denote the set of all bounded linear operators on $X$ into itself. If $X$ is any Banach space and $T \in B(X)$ then the adjoint $T^{*}$ of $T$ is a bounded linear operator on the dual $X^{*}$ of $X$ defined by $\left(T^{*} f\right)(x)=f(T x)$ for all $f \in X^{*}$ and $x \in X$.

Let $X \neq\{\theta\}$ be a nontrivial complex normed space and $T: D(T) \rightarrow X$ a linear operator defined on a subspace $D(T) \subseteq X$. We do not assume that $D(T)$ is dense in $X$ or that $T$ has closed graph $\{(x, T x): x \in D(T)\} \subseteq X \times X$. By the statement " $T$ is invertible," it is meant that there exists a bounded linear operator $S: R(T) \rightarrow X$ for which $S T=I$ on $D(T)$ and $\overline{R(T)}=X$, such that $S=T^{-1}$ is necessarily uniquely determined and linear; the boundedness of $S$ means that $T$ must be bounded below, in the sense that there is $M>0$ for which $\|T x\| \geq M\|x\|$ for all $x \in D(T)$. Associated with each complex number, $\alpha$ is the perturbed operator

$$
T_{\alpha}=\alpha I-T
$$

defined on the same domain $D(T)$ as $T$. The spectrum $\sigma(T, X)$ consists of those $\alpha \in \mathbb{C}$, the complex field, for which $T_{\alpha}$ is not invertible, and the resolvent is the mapping from the complement $\sigma(T, X)$ of the spectrum into the algebra of bounded linear operators on $X$ defined by $\alpha \mapsto T_{\alpha}^{-1}$.

\section{The Subdivisions of Spectrum}

In this section, we define the parts of spectrum called point spectrum, continuous spectrum, residual spectrum, approximate point spectrum, defect spectrum, and compression spectrum. There are many different ways to subdivide the spectrum of a bounded linear operator. Some of them are motivated by applications to physics, in particular, quantum mechanics.

2.1. The Point Spectrum, Continuous Spectrum, and Residual Spectrum. The name resolvent is appropriate since $T_{\alpha}^{-1}$ helps to solve the equation $T_{\alpha} x=y$. Thus, $x=T_{\alpha}^{-1} y$ provided that $T_{\alpha}^{-1}$ exists. More importantly, the investigation of properties of $T_{\alpha}^{-1}$ will be basic for an understanding of the operator $T$ itself. Naturally, many properties of $T_{\alpha}$ and $T_{\alpha}^{-1}$ depend on $\alpha$, and the spectral theory is concerned with those properties. 
For instance, we are interested in the set of all $\alpha$ 's in the complex plane such that $T_{\alpha}^{-1}$ exists. Boundedness of $T_{\alpha}^{-1}$ is another property that will be essential. We will also ask for what $\alpha$ 's the domain of $T_{\alpha}^{-1}$ is dense in $X$, to name just a few aspects. A regular value $\alpha$ of $T$ is a complex number such that $T_{\alpha}^{-1}$ exists and is bounded and whose domain is dense in $X$. For our investigation of $T, T_{\alpha}$, and $T_{\alpha}^{-1}$, we need some basic concepts in the spectral theory which are given, as follows (see [1, pages 370-371]).

The resolvent set $\rho(T, X)$ of $T$ is the set of all regular values $\alpha$ of $T$. Furthermore, the spectrum $\sigma(T, X)$ is partitioned into the following three disjoint sets.

The point (discrete) spectrum $\sigma_{p}(T, X)$ is the set such that $T_{\alpha}^{-1}$ does not exist. An $\alpha \in \sigma_{p}(T, X)$ is called an eigenvalue of $T$.

The continuous spectrum $\sigma_{c}(T, X)$ is the set such that $T_{\alpha}^{-1}$ exists and is unbounded, and the domain of $T_{\alpha}^{-1}$ is dense in $X$.

The residual spectrum $\sigma_{r}(T, X)$ is the set such that $T_{\alpha}^{-1}$ exists (and may be bounded or not) but the domain of $T_{\alpha}^{-1}$ is not dense in $X$.

Therefore, these three subspectra form a disjoint subdivision

$$
\sigma(T, X)=\sigma_{p}(T, X) \cup \sigma_{c}(T, X) \cup \sigma_{r}(T, X) .
$$

To avoid trivial misunderstandings, let us say that some of the sets defined above may be empty. This is an existence problem which we will have to discuss. Indeed, it is well known that $\sigma_{c}(T, X)=\sigma_{r}(T, X)=\emptyset$ and the spectrum $\sigma(T, X)$ consists of only the set $\sigma_{p}(T, X)$ in the finite-dimensional case.

\subsection{The Approximate Point Spectrum, Defect Spectrum, and} Compression Spectrum. In this subsection, following Appell et al. [2], we define three more subdivisions of the spectrum called approximate point spectrum, defect spectrum, and compression spectrum.

Given a bounded linear operator $T$ in a Banach space $X$, we call a sequence $\left(x_{k}\right)$ in $X$ a Weyl sequence for $T$ if $\left\|x_{k}\right\|=1$ and $\left\|T x_{k}\right\| \rightarrow 0$, as $k \rightarrow \infty$. Then, the approximate point spectrum $\sigma_{\text {ap }}(T, X)$ of $T$ is defined by

$$
\begin{aligned}
\sigma_{\text {ap }} & (T, X) \\
& :=\{\alpha \in \mathbb{C}: \text { there exists a Weyl sequence for } \alpha I-T\} .
\end{aligned}
$$

Moreover, the subspectrum

$$
\sigma_{\delta}(T, X):=\{\alpha \in \mathbb{C}: \alpha I-T \text { is not surjective }\}
$$

is called the defect spectrum of $T$.

The two subspectra given by (4) and (5) form a (not necessarily disjoint) subdivision

$$
\sigma(T, X)=\sigma_{\text {ap }}(T, X) \cup \sigma_{\delta}(T, X)
$$

of the spectrum. There is another subspectrum,

$$
\sigma_{\text {co }}(T, X)=\{\alpha \in \mathbb{C}: \overline{R(\alpha I-T)} \neq X\}
$$

which is often called compression spectrum in the literature. The compression spectrum gives rise to another (not necessarily disjoint) decomposition

$$
\sigma(T, X)=\sigma_{\text {ap }}(T, X) \cup \sigma_{\text {co }}(T, X)
$$

of the spectrum. Clearly, $\sigma_{p}(T, X) \subseteq \sigma_{\text {ap }}(T, X)$ and $\sigma_{\text {co }}(T, X) \subseteq$ $\sigma_{\delta}(T, X)$. Moreover, comparing these subspectra with those in (3), we note that

$$
\begin{gathered}
\sigma_{r}(T, X)=\sigma_{\mathrm{co}}(T, X) \backslash \sigma_{p}(T, X), \\
\sigma_{c}(T, X)=\sigma(T, X) \backslash\left[\sigma_{p}(T, X) \cup \sigma_{\mathrm{co}}(T, X)\right] .
\end{gathered}
$$

Sometimes it is useful to relate the spectrum of a bounded linear operator to that of its adjoint. Building on classical existence and uniqueness, results for linear operator equations in Banach spaces and their adjoints are also useful.

Proposition 1 (see [2, Proposition 1.3, page 28]). Spectrum and subspectrum of an operator $T \in B(X)$ and its adjoint $T^{*} \in$ $B\left(X^{*}\right)$ are related by the following relations:

(a) $\sigma\left(T^{*}, X^{*}\right)=\sigma(T, X)$

(b) $\sigma_{c}\left(T^{*}, X^{*}\right) \subseteq \sigma_{a p}(T, X)$,

(c) $\sigma_{a p}\left(T^{*}, X^{*}\right)=\sigma_{\delta}(T, X)$,

(d) $\sigma_{\delta}\left(T^{*}, X^{*}\right)=\sigma_{a p}(T, X)$,

(e) $\sigma_{p}\left(T^{*}, X^{*}\right)=\sigma_{c o}(T, X)$,

(f) $\sigma_{\mathrm{co}}\left(T^{*}, X^{*}\right) \supseteq \sigma_{p}(T, X)$,

(g) $\sigma(T, X)=\sigma_{a p}(T, X) \cup \sigma_{p}\left(T^{*}, X^{*}\right)=\sigma_{p}(T, X) \cup$ $\sigma_{a p}\left(T^{*}, X^{*}\right)$.

The relations (c)-(f) show that the approximate point spectrum is in a certain sense dual to the defect spectrum, and the point spectrum is dual to the compression spectrum. The equality (g) implies, in particular, that $\sigma(T, X)=\sigma_{\text {ap }}(T, X)$ if $X$ is a Hilbert space and $T$ is normal. Roughly speaking, this shows that normal (in particular, self-adjoint) operators on the Hilbert spaces are most similar to matrices in finite dimensional spaces (see [2]).

2.3. Goldberg's Classification of Spectrum. If $X$ is a Banach space and $T \in B(X)$, then there are three possibilities for $R(T)$ :
(A) $R(T)=X$,
(B) $R(T) \neq \overline{R(T)}=X$,
(C) $\overline{R(T)} \neq X$,

and

(1) $T^{-1}$ exists and is continuous,

(2) $T^{-1}$ exists but is discontinuous,

(3) $T^{-1}$ does not exist. 
If these possibilities are combined in all possible ways, nine different states are created. These are labelled by: $A_{1}, A_{2}$, $A_{3}, B_{1}, B_{2}, B_{3}, C_{1}, C_{2}$, and $C_{3}$. If an operator is in state $C_{2}$, for example, then $\overline{R(T)} \neq X$ and $T^{-1}$ exists but is discontinuous (see [3]). Figure 1 due to Wenger [4] may be useful for the readers.

If $\alpha$ is a complex number such that $T_{\alpha} \in A_{1}$ or $T_{\alpha} \in$ $B_{1}$, then $\alpha \in \rho(T, X)$. All scalar values of $\alpha$ not in $\rho(T, X)$ comprise the spectrum of $T$. The further classification of $\sigma(T, X)$ gives rise to the fine spectrum of $T$. That is, $\sigma(T, X)$ can be divided into the subsets $A_{2} \sigma(T, X)=\emptyset, A_{3} \sigma(T, X)$, $B_{2} \sigma(T, X), B_{3} \sigma(T, X), C_{1} \sigma(T, X), C_{2} \sigma(T, X)$, and $C_{3} \sigma(T, X)$. For example, if $T_{\alpha}$ is in a given state, $C_{2}$ (say), then we write $\alpha \in C_{2} \sigma(T, X)$.

By the definitions given above, we can illustrate subdivision (3) in Table 1.

Observe that the case in the first row and the second column cannot occur in a Banach space $X$, by the closed graph theorem. If we are not in the third column, that is, if $\alpha$ is not an eigenvalue of $T$, we may always consider the resolvent operator $T_{\alpha}^{-1}$ (on a possibly "thin" domain of definition) as "algebraic inverse" of $\alpha I-T$.

By a sequence space, we understand a linear subspace of the space $\omega=\mathbb{C}^{\mathbb{N}}$ of all complex sequences which contain $\phi$, the set of all finitely nonzero sequences, where $\mathbb{N}=$ $\{0,1,2, \ldots\}$. We write $\ell_{\infty}, c, c_{0}$, and $b v$ for the spaces of all bounded, convergent, null, and bounded variation sequences which are the Banach spaces with the sup-norm $\|x\|_{\infty}=$ $\sup _{k \in \mathbb{N}}\left|x_{k}\right|$ and $\|x\|_{b v}=\sum_{k=0}^{\infty}\left|x_{k}-x_{k+1}\right|$, respectively, while $\phi$ is not a Banach space with respect to any norm. Also by $\ell_{p}$, we denote the space of all $p$-absolutely summable sequences which is a Banach space with the norm $\|x\|_{p}=$ $\left(\sum_{k=0}^{\infty}\left|x_{k}\right|^{p}\right)^{1 / p}$, where $1 \leq p<\infty$.

Let $\mu$ and $\nu$ be two sequence spaces, and let $A=\left(a_{n k}\right)$ be an infinite matrix of complex numbers $a_{n k}$, where $k, n \in \mathbb{N}$. Then, we say that $A$ defines a matrix transformation from $\mu$ into $\nu$, and we denote it by writing $A: \mu \rightarrow \nu$ if for every sequence $x=\left(x_{k}\right) \in \mu$, the sequence $A x=\left\{(A x)_{n}\right\}$, the $A$ transform of $x$, is in $v$, where

$$
(A x)_{n}=\sum_{k=0}^{\infty} a_{n k} x_{k} \quad \text { for each } n \in \mathbb{N} .
$$

By $(\mu: \nu)$, we denote the class of all matrices $A$ such that $A: \mu \rightarrow \nu$. Thus, $A \in(\mu: \nu)$ if and only if the series on the right side of (10) converges for each $n \in \mathbb{N}$ and each $x \in \mu$, and we have $A x=\left\{(A x)_{n}\right\}_{n \in \mathbb{N}} \in \nu$ for all $x \in \mu$.

Throughout this paper, let $\lambda=\left(\lambda_{k}\right)$ be a strictly increasing sequence of positive reals tending to infinity; that is,

$$
0<\lambda_{0}<\lambda_{1}<\lambda_{2}<\cdots, \quad \lim _{k \rightarrow \infty} \lambda_{k}=\infty .
$$

Following Mursaleen and Noman [20], we define the matrix $\Lambda=\left(\lambda_{n k}\right)$ of weighted mean relative to the sequence $\lambda$ by

$$
\lambda_{n k}= \begin{cases}\frac{\lambda_{k}-\lambda_{k-1}}{\lambda_{n}}, & 0 \leq k \leq n \\ 0, & k>n\end{cases}
$$

for all $k, n \in \mathbb{N}$. It is easy to show that the matrix $\Lambda$ is regular and is reduced, in the special case $\lambda_{k}=k+1$ for all $k \in \mathbb{N}$, to

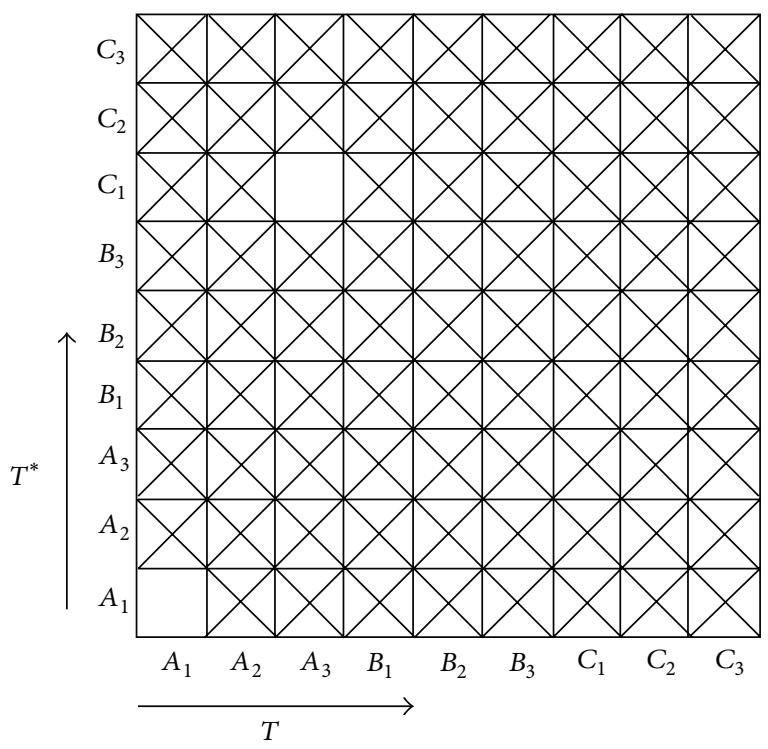

FIgURE 1: State diagram for $B(X)$ and $B\left(X^{*}\right)$ for a non-reflective Banach space $X$.

the matrix $C_{1}$ of Cesàro mean of order one. Introducing the concept of $\Lambda$-strong convergence, several results on $\Lambda$-strong convergence of numerical sequences and Fourier series were given by Móricz [21]. Since we have

$$
Q_{n}=\sum_{k=0}^{n} q_{k}=\lambda_{n}, \quad r_{n k}=\frac{q_{k}}{Q_{n}}=\frac{\lambda_{k}-\lambda_{k-1}}{\lambda_{n}}=\lambda_{n k}
$$

in the special case $q_{k}=\lambda_{k}-\lambda_{k-1}$ for all $k \in \mathbb{N}$, the matrix $\Lambda$ is also reduced to the Riesz means $R^{q}=\left(r_{n k}\right)$ with respect to the sequence $q=\left(q_{k}\right)$. We say that a sequence $x=\left(x_{k}\right) \in \omega$ is $\lambda$-convergent if $\Lambda x \in c$. In particular, we say that $x$ is a $\lambda$ null sequence if $\Lambda x \in c_{0}$ and we say that $x$ is $\lambda$-bounded if $\Lambda x \in \ell_{\infty}$.

Lemma 2 (see [22, Theorem 1.3.6, page 6]). The matrix $A=$ $\left(a_{n k}\right)$ gives rise to a bounded linear operator $T \in B(c)$ from $c$ to itself if and only if

(1) the rows of $A$ are in $\ell_{1}$ and their $\ell_{1}$ norms are bounded;

(2) the columns of $A$ are in $c$;

(3) the sequence of row sums of $A$ is in $c$.

The operator norm of $T$ is the supremum of the $\ell_{1}$ norms of the rows.

Corollary 3. $\Lambda: c \rightarrow c$ is a bounded linear operator with the norm $\|\Lambda\|_{(c: c)}=1$.

Lemma 4 (see [22, Example 8.4.5.A, page 129]). The matrix $A=\left(a_{n k}\right)$ gives rise to a bounded linear operator $T \in B\left(c_{0}\right)$ from $c_{0}$ to itself if and only if

(1) the rows of $A$ are in $\ell_{1}$ and their $\ell_{1}$ norms are bounded,

(2) the columns of $A$ are in $c_{0}$. 
TABLE 1: Subdivision of spectrum of a linear operator.

\begin{tabular}{|c|c|c|c|c|}
\hline & & $\begin{array}{c}1 \\
T_{\alpha}^{-1} \text { exists } \\
\text { and is bounded }\end{array}$ & $\begin{array}{c}2 \\
T_{\alpha}^{-1} \text { exists } \\
\text { and is unbounded }\end{array}$ & $\begin{array}{c}3 \\
T_{\alpha}^{-1} \\
\text { does not exist }\end{array}$ \\
\hline \multirow{2}{*}{ A } & \multirow{2}{*}{$R(\alpha I-T)=X$} & \multirow{2}{*}{$\alpha \in \rho(T, X)$} & \multirow{2}{*}{-} & $\alpha \in \sigma_{p}(T, X)$ \\
\hline & & & & $\alpha \in \sigma_{\text {ap }}(T, X)$ \\
\hline \multirow{3}{*}{ B } & \multirow{3}{*}{$\overline{R(\alpha I-T)}=X$} & \multirow{3}{*}{$\alpha \in \rho(T, X)$} & $\alpha \in \sigma_{c}(T, X)$ & $\alpha \in \sigma_{p}(T, X)$ \\
\hline & & & $\alpha \in \sigma_{\mathrm{ap}}(T, X)$ & $\alpha \in \sigma_{\mathrm{ap}}(T, X)$ \\
\hline & & & $\alpha \in \sigma_{\delta}(T, X)$ & $\alpha \in \sigma_{\delta}(T, X)$ \\
\hline \multirow{4}{*}{$\mathrm{C}$} & \multirow{4}{*}{$\overline{R(\alpha I-T)} \neq X$} & $\alpha \in \sigma_{r}(T, X)$ & $\alpha \in \sigma_{r}(T, X)$ & $\alpha \in \sigma_{p}(T, X)$ \\
\hline & & \multirow{2}{*}{$\alpha \in \sigma_{\delta}(T, X)$} & $\alpha \in \sigma_{\mathrm{ap}}(T, X)$ & $\alpha \in \sigma_{\mathrm{ap}}(T, X)$ \\
\hline & & & $\alpha \in \sigma_{\delta}(T, X)$ & $\alpha \in \sigma_{\delta}(T, X)$ \\
\hline & & $\alpha \in \sigma_{\mathrm{co}}(T, X)$ & $\alpha \in \sigma_{\mathrm{co}}(T, X)$ & $\alpha \in \sigma_{\mathrm{co}}(T, X)$ \\
\hline
\end{tabular}

The operator norm of $T$ is the supremum of the $\ell_{1}$ norms of the rows.

Corollary 5. $\Lambda: c_{0} \rightarrow c_{0}$ is a bounded linear operator with the norm $\|\Lambda\|_{\left(c_{0}: c_{0}\right)}=1$.

We give a short survey concerned with the spectrum of the linear operators defined by some triangle matrices over certain sequence spaces. Wenger [4] examined the fine spectrum of the integer power of the Cesàro operator in $c$ and Rhoades [5] generalized this result to the weighted mean methods. The fine spectrum of the operator on the sequence space $\ell_{p}$ was studied by González [23], where $1<p<\infty$. The spectrum of the Cesàro operator on the sequence spaces $c_{0}$ and $b v$ were also investigated by Reade [6], Akhmedov and Başar [7], and Okutoyi [8], respectively. The fine spectrum of the Rhaly operators on the sequence spaces $c_{0}$ and $c$ were examined by Yıldırım [9]. Furthermore, Coşkun [10] has studied the spectrum and fine spectrum for $p$-Cesàro operator acting on the space $c_{0}$. Besides, de Malafosse [11] and Altay and Başar [12], respectively, studied the spectrum and the fine spectrum of the difference operator on the sequence spaces $s_{r}$ and $c_{0}, c$, where $s_{r}$ denotes the Banach space of all sequences $x=\left(x_{k}\right)$ normed by $\|x\|_{s_{r}}=\sup _{k \in \mathbb{N}}\left|x_{k}\right| / r^{k}$, $(r>0)$. Altay and Karakuş [24] determined the fine spectrum of the Zweier matrix which is a band matrix as an operator over the sequence spaces $\ell_{1}$ and $b v$. In 2010, Srivastava and Kumar [16] determined the spectra and the fine spectra of the double sequential band matrix $\Delta_{v}$ on $\ell_{1}$, where $\Delta_{v}$ is defined by $\left(\Delta_{\gamma}\right)_{n n}=\nu_{n}$ and $\left(\Delta_{\nu}\right)_{n+1, n}=-\nu_{n}$ for all $n \in \mathbb{N}$, under certain conditions on the sequence $v=\left(\nu_{k}\right)$ and they have just generalized these results by the double sequential band matrix $\Delta_{u v}$ defined by $\Delta_{u v} x=\left(u_{n} x_{n}+v_{n-1} x_{n-1}\right)_{n \in \mathbb{N}}$ for all $n \in \mathbb{N}$ (see [18]). Altun [25] studied the fine spectra of the Toeplitz operators, which are represented by upper and lower triangular $n$-band infinite matrices, over the sequence spaces $c_{0}$ and $c$. Later, Karakaya and Altun determined the fine spectra of upper triangular double-band matrices over the sequence spaces $c_{0}$ and $c$, in [26]. Quite recently, Akhmedov and El-Shabrawy [15] obtained the fine spectrum of the double sequential band matrix $\Delta_{a, b}$, defined as a doubleband matrix with the convergent sequences $\widetilde{a}=\left(a_{k}\right)$ and $\widetilde{b}=\left(b_{k}\right)$ having certain properties, over the sequence space $c$. The fine spectrum with respect to Goldberg's classification of the operator $B(r, s, t)$ defined by a triple band matrix over the sequence spaces $\ell_{p}$ and $b v_{p}$ with $1<p<\infty$ has recently been studied by Furkan et al. [14]. Quite recently, Karaisa and Başar [19] have determined the fine spectrum of the upper triangular triple band matrix $B^{\prime}(r, s, t)$ over the sequence space $\ell_{p}$, where $0<p<\infty$. At this stage, Table 2 may be useful.

In this work, our purpose is to determine the fine spectrum of the operator $\Lambda$ over the sequence spaces $c_{0}$ and $c$ with respect to Goldberg's classification. Additionally, we give the approximate point spectrum, defect spectrum, and compression spectrum of the matrix operator $\Lambda$ over the spaces $c_{0}$ and $c$. Finally, we state and prove a Mercerian theorem.

\section{The Fine Spectrum of the Operator $\Lambda$ on the Sequence Space $c_{0}$}

In this section, we examine the spectrum, the point spectrum, the continuous spectrum, the residual spectrum, the fine spectrum, the approximate point spectrum, the defect spectrum, and the compression spectrum of the operator $\Lambda$ on the sequence space $c_{0}$. For simplicity in the notation, we write throughout that $c_{n}=\left(\lambda_{n}-\lambda_{n-1}\right) / \lambda_{n}$ for all $n \in \mathbb{N}$ and we use this abbreviation with other letters.

Theorem 6. $\sigma\left(\Lambda, c_{0}\right) \subseteq\{\alpha \in \mathbb{C}:|2 \alpha-1| \leq 1\}$.

Proof. Let $|2 \alpha-1|>1$. Since $\Lambda-\alpha I$ is triangle, $(\Lambda-\alpha I)^{-1}$ exists, and solving the matrix equation $(\Lambda-\alpha I) x=y$ for $x$ in terms of $y$ gives the matrix $(\Lambda-\alpha I)^{-1}=B=\left(b_{n k}\right)$, where

$$
b_{n k}= \begin{cases}\frac{(-1)^{n-k}\left(\lambda_{k}-\lambda_{k-1}\right)}{\lambda_{n} \alpha^{2} \prod_{j=k}^{n}\left(c_{j} / \alpha-1\right)}, & 0 \leq k \leq n-1, \\ \frac{\lambda_{n}}{\lambda_{n}-\lambda_{n-1}-\alpha \lambda_{n}}, & k=n, \\ 0, & k>n,\end{cases}
$$


TABLE 2: Spectrum and fine spectrum of some triangle matrices in certain sequence spaces.

\begin{tabular}{|c|c|c|c|c|}
\hline$\sigma(A, \lambda)$ & $\sigma_{p}(A, \lambda)$ & $\sigma_{c}(A, \lambda)$ & $\sigma_{r}(A, \lambda)$ & Refer to \\
\hline$\sigma\left(C_{1}^{p}, c\right)$ & - & - & - & [4] \\
\hline$\sigma(W, c)$ & - & - & - & [5] \\
\hline$\sigma\left(C_{1}, c_{0}\right)$ & - & - & - & {$[6]$} \\
\hline$\sigma\left(C_{1}, c_{0}\right)$ & $\sigma_{p}\left(C_{1}, c_{0}\right)$ & $\sigma_{c}\left(C_{1}, c_{0}\right)$ & $\sigma_{r}\left(C_{1}, c_{0}\right)$ & [7] \\
\hline$\sigma\left(C_{1}, b v\right)$ & - & - & - & {$[8]$} \\
\hline$\sigma\left(R, c_{0}\right)$ & $\sigma_{p}\left(R, c_{0}\right)$ & $\sigma_{c}\left(R, c_{0}\right)$ & $\sigma_{r}\left(R, c_{0}\right)$ & [9] \\
\hline$\sigma(R, c)$ & $\sigma_{p}(R, c)$ & $\sigma_{c}(R, c)$ & $\sigma_{r}(R, c)$ & [9] \\
\hline$\sigma\left(C_{1}^{p}, c_{0}\right)$ & - & - & - & [10] \\
\hline$\sigma\left(\Delta, s_{r}\right)$ & - & - & - & [11] \\
\hline$\sigma\left(\Delta, c_{0}\right)$ & - & - & - & [11] \\
\hline$\sigma(\Delta, c)$ & - & - & - & [11] \\
\hline$\sigma\left(\Delta^{(1)}, c\right)$ & $\sigma_{p}\left(\Delta^{(1)}, c\right)$ & $\sigma_{c}\left(\Delta^{(1)}, c\right)$ & $\sigma_{r}\left(\Delta^{(1)}, c\right)$ & [12] \\
\hline$\sigma\left(\Delta^{(1)}, c_{0}\right)$ & $\sigma_{p}\left(\Delta^{(1)}, c_{0}\right)$ & $\sigma_{c}\left(\Delta^{(1)}, c_{0}\right)$ & $\sigma_{r}\left(\Delta^{(1)}, c_{0}\right)$ & {$[12]$} \\
\hline$\sigma\left(B(r, s), \ell_{p}\right)$ & $\sigma_{p}\left(B(r, s), \ell_{p}\right)$ & $\sigma_{c}\left(B(r, s), \ell_{p}\right)$ & $\sigma_{r}\left(B(r, s), \ell_{p}\right)$ & [13] \\
\hline$\sigma\left(B(r, s), b v_{p}\right)$ & $\sigma_{p}\left(B(r, s), b v_{p}\right)$ & $\sigma_{c}\left(B(r, s), b v_{p}\right)$ & $\sigma_{r}\left(B(r, s), b v_{p}\right)$ & {$[13]$} \\
\hline$\sigma\left(B(r, s, t), \ell_{p}\right)$ & $\sigma_{p}\left(B(r, s, t), \ell_{p}\right)$ & $\sigma_{c}\left(B(r, s, t), \ell_{p}\right)$ & $\sigma_{r}\left(B(r, s, t), \ell_{p}\right)$ & {$[14]$} \\
\hline$\sigma\left(B(r, s, t), b v_{p}\right)$ & $\sigma_{p}\left(B(r, s, t), b v_{p}\right)$ & $\sigma_{c}\left(B(r, s, t), b v_{p}\right)$ & $\sigma_{r}\left(B(r, s, t), b v_{p}\right)$ & [14] \\
\hline$\sigma\left(\Delta_{a, b}, c\right)$ & $\sigma_{p}\left(\Delta_{a, b}, c\right)$ & $\sigma_{c}\left(\Delta_{a, b}, c\right)$ & $\sigma_{r}\left(\Delta_{a, b}, c\right)$ & [15] \\
\hline$\sigma\left(\Delta_{v}, \ell_{1}\right)$ & $\sigma_{p}\left(\Delta_{v}, \ell_{1}\right)$ & $\sigma_{c}\left(\Delta_{v}, \ell_{1}\right)$ & $\sigma_{r}\left(\Delta_{v}, \ell_{1}\right)$ & [16] \\
\hline$\sigma\left(\Delta_{u v}^{2}, c_{0}\right)$ & $\sigma_{p}\left(\Delta_{u v}^{2}, c_{0}\right)$ & $\sigma_{c}\left(\Delta_{u v}^{2}, c_{0}\right)$ & $\sigma_{r}\left(\Delta_{u v}^{2}, c_{0}\right)$ & [17] \\
\hline$\sigma\left(\Delta_{u v}, \ell_{1}\right)$ & $\sigma_{p}\left(\Delta_{u v}, \ell_{1}\right)$ & $\sigma_{c}\left(\Delta_{u v}, \ell_{1}\right)$ & $\sigma_{r}\left(\Delta_{u v}, \ell_{1}\right)$ & [18] \\
\hline$\sigma\left(B^{\prime}(r, s, t), \ell_{p}\right)$ & $\sigma_{p}\left(B^{\prime}(r, s, t), \ell_{p}\right)$ & $\sigma_{c}\left(B^{\prime}(r, s, t), \ell_{p}\right)$ & $\sigma_{r}\left(B^{\prime}(r, s, t), \ell_{p}\right)$ & [19] \\
\hline
\end{tabular}

for all $k, n \in \mathbb{N}$. Thus, we observe that

$$
\left\|(\Lambda-\alpha I)^{-1}\right\|_{\left(c_{0}: c_{0}\right)}=\sup _{n \in \mathbb{N}} \sum_{k=0}^{\infty}\left|b_{n k}\right| .
$$

The inequality $|2 \alpha-1|>1$ is equivalent to $\gamma>-1$, where $-(1 / \alpha)=\gamma+i \beta$. For all $\alpha \in \mathbb{C}$,

$$
\left|1-\frac{c_{j}}{\alpha}\right|=\left|1+(\gamma+i \beta) c_{j}\right| \geq 1+\gamma c_{j}
$$

holds for all $j \in \mathbb{N}$. So, $1 /\left|1-\left(c_{j} / \alpha\right)\right| \leq 1 /\left(1+\gamma c_{j}\right)$.

Firstly we take $-1<\gamma<0$. Since $0<c_{j} \leq 1$, we have $1+\gamma \leq 1+\gamma c_{j}<1$. Therefore $1 /\left(1+\gamma c_{j}\right)<1 /(1+\gamma)$ and $1<1 /(1+\gamma)<\infty$ for $0<1+\gamma<1$.

$$
\sum_{k=0}^{\infty}\left|b_{n k}\right|=\sum_{k=0}^{n}\left|b_{n k}\right|<\frac{\lambda_{n-1}}{\lambda_{n}|\alpha|^{2}(1+\gamma)^{n+1}}+\frac{1}{|\alpha|(1+\gamma)}<\infty .
$$

Secondly we get $0 \leq \gamma$. Since $1<1+\gamma c_{j} \leq 1+\gamma, 1 /(1+$ $\left.\gamma c_{j}\right)<1$. So,

$$
\sum_{k=0}^{\infty}\left|b_{n k}\right|=\sum_{k=0}^{n}\left|b_{n k}\right|<\frac{\lambda_{n-1}}{\lambda_{n}|\alpha|^{2}}+\frac{1}{|\alpha|}<\infty .
$$

Therefore, we have

$$
\left\|(\Lambda-\alpha I)^{-1}\right\|_{\left(c_{0}: c_{0}\right)}=\sup _{n \in \mathbb{N}} \sum_{k=0}^{n}\left|b_{n k}\right|<\infty,
$$

that is, $(\Lambda-\alpha I)^{-1} \in\left(c_{0}: c_{0}\right)$. But for $|2 \alpha-1| \leq 1$,

$$
\left\|(\Lambda-\alpha I)^{-1}\right\|_{\left(c_{0}: c_{0}\right)}=\infty \text {, }
$$

that is, $(\Lambda-\alpha I)^{-1}$ is not in $B\left(c_{0}\right)$. This completes the proof.

Theorem 7. Define $\mu$ and $\eta$ by $\mu=\limsup _{j \rightarrow \infty} c_{j}$ and $\eta=$ $\liminf _{j \rightarrow \infty} c_{j}$. Then,

$$
\begin{aligned}
& \left\{\alpha \in \mathbb{C}:\left|\alpha-\frac{1}{2-\mu}\right| \leq \frac{1-\mu}{2-\mu}\right\} \cup S \subseteq \sigma\left(\Lambda, c_{0}\right), \\
& \text { where } S=\overline{\left\{c_{j}: j \in \mathbb{N}\right\} .}
\end{aligned}
$$

Proof. Let $|\alpha-1 /(2-\mu)|<(1-\mu) /(2-\mu)$ and $\alpha \neq c_{j}$ for any $j \in \mathbb{N}$. Then,

$$
\begin{aligned}
1-\frac{c_{j}}{\alpha} & =\frac{\lambda_{j} \lambda_{j-1}}{\lambda_{j} \lambda_{j-1}}-\frac{c_{j}}{\alpha} \\
& =\frac{\lambda_{j-1}}{\lambda_{j}}\left[\frac{\lambda_{j}}{\lambda_{j-1}}-\frac{\lambda_{j}-\lambda_{j-1}}{\lambda_{j-1}}+\left(1-\frac{1}{\alpha}\right) \frac{\lambda_{j}-\lambda_{j-1}}{\lambda_{j-1}}\right] \\
& =\frac{\lambda_{j-1}}{\lambda_{j}}\left[1+\left(1-\frac{1}{\alpha}\right) \frac{\lambda_{j}-\lambda_{j-1}}{\lambda_{j-1}}\right] .
\end{aligned}
$$


So we have,

$$
\left|b_{n k}\right|=\frac{\lambda_{k}-\lambda_{k-1}}{\lambda_{k-1}|\alpha|^{2} \prod_{j=k}^{n}\left|1+(1-1 / \alpha)\left(\left(\lambda_{j}-\lambda_{j-1}\right) / \lambda_{j-1}\right)\right|} .
$$

Note that $\left|1+\left(1-\alpha^{-1}\right)\left(\lambda_{j}-\lambda_{j-1}\right) / \lambda_{j-1}\right| \leq 1$ if and only if

$$
\left[1+(1+\gamma) \frac{\lambda_{j}-\lambda_{j-1}}{\lambda_{j-1}}\right]^{2}+\left(\beta \frac{\lambda_{j}-\lambda_{j-1}}{\lambda_{j-1}}\right)^{2} \leq 1
$$

where $-\alpha^{-1}=\gamma+i \beta$. So, one can see that

$$
2(1+\gamma) \frac{\lambda_{j}-\lambda_{j-1}}{\lambda_{j-1}}+\left[(1+\gamma)^{2}+\beta^{2}\right]\left(\frac{\lambda_{j}-\lambda_{j-1}}{\lambda_{j-1}}\right)^{2} \leq 0,
$$

which is equivalent to the inequality

$$
2(1+\gamma)+\left[(1+\gamma)^{2}+\beta^{2}\right]\left(\frac{\lambda_{j}-\lambda_{j-1}}{\lambda_{j-1}}\right) \leq 0 .
$$

For inequality (26) to be true for all sufficiently large $j$, it is sufficient to have

$$
\limsup _{j \rightarrow \infty}\left[2(1+\gamma)+\left[(1+\gamma)^{2}+\beta^{2}\right] \frac{\lambda_{j}-\lambda_{j-1}}{\lambda_{j-1}}\right]<0 .
$$

We can write $\left(\lambda_{j}-\lambda_{j-1}\right) / \lambda_{j-1}=\lambda_{j}\left(\lambda_{j}-\lambda_{j-1}\right) /\left(\lambda_{j} \lambda_{j-1}\right)$ and $\lambda_{j} / \lambda_{j-1}=1 /\left(1-c_{j}\right)$. Therefore,

$$
\begin{gathered}
\frac{\lambda_{j}-\lambda_{j-1}}{\lambda_{j-1}}=\frac{c_{j}}{1-c_{j}}, \\
\limsup _{j \rightarrow \infty} \frac{\lambda_{j}-\lambda_{j-1}}{\lambda_{j-1}}=\frac{\mu}{1-\mu},
\end{gathered}
$$

since the function $g$ defined by $g(x)=x /(1-x)$ is monotone increasing in $x$ for $0<x<1$.

For (27) to be true for all sufficiently large $j$, it is sufficient to have $\mu$ satisfying

$$
2(1+\gamma)+\left[(1+\gamma)^{2}+\beta^{2}\right] \frac{\mu}{1-\mu}<0
$$

which is equivalent to

$$
\left|\alpha-\frac{1}{2-\mu}\right|<\frac{1-\mu}{2-\mu} .
$$

Therefore, for all $n \geq N$, for some fixed $N$,

$$
\begin{aligned}
\sum_{k=N}^{n-1}\left|b_{n k}\right| & =\sum_{k=N}^{n-1} \frac{\lambda_{k}-\lambda_{k-1}}{\lambda_{k-1}|\alpha|^{2} \prod_{j=k}^{n}\left|1+(1-1 / \alpha)\left(\left(\lambda_{j}-\lambda_{j-1}\right) / \lambda_{j-1}\right)\right|} \\
& \geq \frac{1}{|\alpha|^{2}} \sum_{k=N}^{n-1} \frac{\lambda_{k}-\lambda_{k-1}}{\lambda_{k-1}}
\end{aligned}
$$

which diverges in the light of (29).

If $\alpha=c_{j}$ for any $j \in \mathbb{N}$, then clearly $\alpha$ lies in the spectrum of $\Lambda$. This completes the proof.
Theorem 8. $\sigma\left(\Lambda, c_{0}\right) \subseteq\{\alpha \in \mathbb{C}:|\alpha-1 /(2-\eta)| \leq(1-\eta) /(2-$ $\eta)\} \cup S$.

Proof. Let $\alpha$ be fixed and satisfy the inequality

$$
\left|\alpha-\frac{1}{2-\eta}\right|>\frac{1-\eta}{2-\eta}
$$

and $\alpha \neq c_{j}$ for any $j \in \mathbb{N}$. We will show that $\alpha \in \rho\left(\Lambda, c_{0}\right)$. From Theorem 6 , we need consider only those values of $\alpha$ satisfying $|2 \alpha-1|>1$; that is, $\gamma>-1$. Under the assumption on $\alpha$, we wish to verify that

$$
\left|1+\left(1-\frac{1}{\alpha}\right) \frac{\lambda_{j}-\lambda_{j-1}}{\lambda_{j-1}}\right|>1
$$

for all sufficiently large $j$. It will be sufficient to show that

$$
\liminf _{j \rightarrow \infty}\left\{2(1+\gamma)+\left[(1+\gamma)^{2}+\beta^{2}\right] \frac{\lambda_{j}-\lambda_{j-1}}{\lambda_{j-1}}\right\}>0
$$

that is,

$$
2(1+\gamma)+\left[(1+\gamma)^{2}+\beta^{2}\right] \frac{\eta}{1-\eta}>0
$$

which is equivalent to (33).

Define the function $f$ by $f(t)=1+2(1+\gamma) t+\left[(1+\gamma)^{2}+\right.$ $\left.\beta^{2}\right] t^{2} . f$ has a minumum at $t_{0}=-(1+\gamma) /\left[(1+\gamma)^{2}+\beta^{2}\right]$. The above inequality is equivalent to $\eta\left(\gamma^{2}+\beta^{2}\right)+2 \gamma>\eta-2$ and is also equivalent to

$$
\frac{\eta}{2(1-\eta)}>-\frac{1+\gamma}{(1+\gamma)^{2}+\beta^{2}}=t_{0}
$$

Therefore, for those values of $\eta$ satisfying (37), $f$ is monotone increasing. Let $\epsilon>0$ and small. Then $f(\eta /(1-\eta)-\epsilon)=$ $f(\eta /(1-\eta))-(2 \epsilon) g(\epsilon)$, where $g(\epsilon)=1+\gamma+\left[(1+\gamma)^{2}+\right.$ $\left.\beta^{2}\right][\eta /(1-\eta)-\epsilon / 2]$. Note that $g(\epsilon)>0$ for small $\epsilon$, since $f$ is monotone increasing for $t>\eta /[2(1-\eta)]$, we will now show that $f(\eta /(1-\eta))>1$. From (37),

$$
\gamma^{2}+\beta^{2}+\frac{2 \gamma}{\eta}>\frac{\eta-2}{\eta}
$$

which is equivalent to

$$
\left|\frac{1}{1-\eta}-\frac{\eta}{\alpha(1-\eta)}\right|>1
$$

But $1 /(1-\eta)=1+\eta /(1-\eta)$, so we have $f(\eta /(1-\eta))=$ $\left|1+\left(1-\alpha^{-1}\right) \eta /(1-\eta)\right|^{2}>1$. Now choose $\epsilon>0$ and so small that $f(\eta /(1-\eta)-\epsilon)=f(\eta /(1-\eta))-2 \epsilon g(\epsilon)=m^{2}>1$. Then, by the definition of $\eta$, there exists an $N$ such that $n>N$ implies 
$\left(\lambda_{n+1}-\lambda_{n}\right) / \lambda_{n}>\eta /(1-\eta)-\epsilon$, so that $f\left(\left(\lambda_{n+1}-\lambda_{n}\right) / \lambda_{n}\right)>$ $f(\eta /(1-\eta)-\epsilon)=m^{2}$. Using $(23)$,

$$
\begin{aligned}
& \frac{\left|b_{n k}\right|}{\left|b_{n+1, k}\right|} \\
& =\frac{\left(\lambda_{k}-\lambda_{k-1}\right) / \lambda_{k-1}|\alpha|^{2} \prod_{j=k}^{n}\left|1+(1-1 / \alpha)\left(\left(\lambda_{j}-\lambda_{j-1}\right) / \lambda_{j-1}\right)\right|}{\left(\lambda_{k}-\lambda_{k-1}\right) / \lambda_{k-1}|\alpha|^{2} \prod_{j=k}^{n+1}\left|1+(1-1 / \alpha)\left(\left(\lambda_{j}-\lambda_{j-1}\right) / \lambda_{j-1}\right)\right|} \\
& =\left|1+\left(1-\frac{1}{\alpha}\right) \frac{\lambda_{n+1}-\lambda_{n}}{\lambda_{n}}\right| \\
& =f\left(\frac{\lambda_{n+1}-\lambda_{n}}{\lambda_{n}}\right)>m^{2}>1,
\end{aligned}
$$

for all $n \geq N$. Therefore $\left\{\left|b_{n k}\right|\right\}$ is monotone decreasing in $n$ for each $k, n>N$, so that $B$ has bounded columns. It remains to show that $B$ has finite norm.

For $\epsilon$ being used, from (29), we can enlarge $N$, if necessary, to ensure that $\left(\lambda_{n}-\lambda_{n-1}\right) / \lambda_{n-1}<\mu /(1-\mu)+1$ for $n \geq N$. From (23),

$$
\begin{aligned}
& \sum_{k=N}^{n-1}\left|b_{n k}\right| \\
& =\sum_{k=N}^{n-1} \frac{\lambda_{k}-\lambda_{k-1}}{\lambda_{k-1}|\alpha|^{2} \prod_{j=k}^{n}\left|1+(1-1 / \alpha)\left(\left(\lambda_{j}-\lambda_{j-1}\right) / \lambda_{j-1}\right)\right|} \\
& \leq \frac{1}{|\alpha|^{2}}\left(\frac{\mu}{1-\mu}+1\right) \sum_{k=N}^{n-1} \frac{1}{\prod_{j=k}^{n}\left|1+(1-1 / \alpha)\left(\left(\lambda_{j}-\lambda_{j-1}\right) / \lambda_{j-1}\right)\right|} \\
& \leq \frac{1}{|\alpha|^{2}}\left(\frac{\mu}{1-\mu}+1\right) \sum_{k=N}^{n-1} m^{-n+k-1}<H,
\end{aligned}
$$

where $H$ is a constant independent of $n$. Further

$$
\begin{aligned}
\left|b_{n n}\right| & =\frac{\lambda_{n}}{|\alpha|\left|\lambda_{n}-\left(\left(\lambda_{n}-\lambda_{n-1}\right) / \alpha\right)\right|} \\
& =\frac{\lambda_{n}}{|\alpha|\left|\lambda_{n-1}+(1-1 / \alpha)\left(\lambda_{n}-\lambda_{n-1}\right)\right|} \\
& =\frac{\lambda_{n} / \lambda_{n-1}}{|\alpha|\left|1+(1-1 / \alpha)\left(\left(\lambda_{n}-\lambda_{n-1}\right) / \lambda_{n-1}\right)\right|} \\
& =\frac{1+\left(\lambda_{n}-\lambda_{n-1}\right) / \lambda_{n-1}}{|\alpha|\left|1+(1-1 / \alpha)\left(\left(\lambda_{n}-\lambda_{n-1}\right) / \lambda_{n-1}\right)\right|} \\
& <\frac{1+\mu /(1-\mu)+1}{|\alpha| m} .
\end{aligned}
$$

Hence, $B$ has a finite norm.

Corollary 9. Let $\delta=\lim _{j \rightarrow \infty} c_{j}$ exist. Then,

$$
\sigma\left(\Lambda, c_{0}\right)=\left\{\alpha \in \mathbb{C}:\left|\alpha-\frac{1}{2-\delta}\right| \leq \frac{1-\delta}{2-\delta}\right\} \cup S
$$

If $T \in B\left(c_{0}\right)$ with the matrix $A$, then it is known that the adjoint operator $T^{*}: c_{0}^{*} \rightarrow c_{0}^{*}$ is dened by the transpose $A^{t}$ of the matrix $A$. It should be noted that the dual space $c_{0}^{*}$ of $c_{0}$ is isometrically isomorphic to the Banach space $\ell_{1}$ of absolutely summable sequences normed by $\|x\|=\sum_{k=0}^{\infty}\left|x_{k}\right|$.

Theorem 10. Let $\delta$ be defined as in Corollary 9. Then, $\sigma_{p}\left(\Lambda^{*}, c_{0}^{*}\right)=\{\alpha \in \mathbb{C}:|\alpha-1 /(2-\delta)|<(1-\delta) /(2-\delta)\} \cup S$.

Proof. Suppose that $\Lambda^{*} x=\alpha x$ for $x \neq \theta$ in $c_{0}^{*} \cong \ell_{1}$. Then, by solving the system of linear equations

$$
\begin{aligned}
x_{1}= & \frac{\lambda_{1}-\lambda_{0}}{\lambda_{0}}\left(1-\frac{1}{\alpha}\right) x_{0}, \\
x_{2}= & \frac{\lambda_{2}-\lambda_{1}}{\lambda_{0}}\left(1-\frac{c_{1}}{\alpha}\right)\left(1-\frac{1}{\alpha}\right) x_{0} \\
& \vdots \\
x_{n}= & \frac{\lambda_{n}-\lambda_{n-1}}{\lambda_{0}}\left(1-\frac{1}{\alpha}\right) x_{0} \prod_{j=1}^{n-1}\left(1-\frac{c_{j}}{\alpha}\right)
\end{aligned}
$$

we can write $x_{n}=\left(\lambda_{n}-\lambda_{n-1}\right) / \lambda_{n-1}\left(1-\alpha^{-1}\right) x_{0} \prod_{j=1}^{n-1}[1+(1-$ $\left.\left.\alpha^{-1}\right)\left(\lambda_{j}-\lambda_{j-1}\right) / \lambda_{j-1}\right]$. Let $|\alpha-1 /(2-\delta)|<(1-\delta) /(2-\delta)$ or $\alpha \in S$ and $u_{n}=\prod_{j=1}^{n-1}\left[1+\left(1-\alpha^{-1}\right)\left(\lambda_{j}-\lambda_{j-1}\right) / \lambda_{j-1}\right]$. One can see that $\left|1+\left(1-\alpha^{-1}\right)\left(\lambda_{j}-\lambda_{j-1}\right) / \lambda_{j-1}\right|<1$ for all sufficiently large $j$ if and only if

$$
\begin{array}{r}
{\left[1+(1+\gamma) \frac{\lambda_{j}-\lambda_{j-1}}{\lambda_{j-1}}\right]^{2}+\left(\beta \frac{\lambda_{j}-\lambda_{j-1}}{\lambda_{j-1}}\right)^{2}<1,} \\
\text { where }-\frac{1}{\alpha}=\gamma+i \beta .
\end{array}
$$

Then, we have, from the discussion in Theorem 7 and the hypothesis on $\alpha$,

$$
\left|\frac{u_{n+1}}{u_{n}}\right|=\left|1+\left(1-\frac{1}{\alpha}\right) \frac{\lambda_{n}-\lambda_{n-1}}{\lambda_{n-1}}\right|<1
$$

for all sufficiently large $n$, so $\sum_{n=0}^{\infty}\left|u_{n}\right|$ is convergent. Since $\left|\left(1-\alpha^{-1}\right)\left(\lambda_{n}-\lambda_{n-1}\right) / \lambda_{n-1} x_{0}\right|$ is bounded, it follows that $\sum_{n=0}^{\infty}\left|x_{n}\right|$ is convergent, so that $\Lambda^{*} x=\alpha x$ has nonzero solutions. Therefore, the proof is completed.

Theorem 11. Let $\delta$ be defined as in Corollary 9. Then

$$
\sigma_{p}\left(\Lambda, c_{0}\right)=\left\{\alpha=c_{n} \in \mathbb{C}: 0 \leq \alpha \leq \frac{\delta}{2-\delta}\right\} \cup\{1\} .
$$

Proof. Let $c_{k}$ be any diagonal entry satisfying $0<c_{k} \leq \delta /(2-$ $\delta)$. Let $j$ be the smallest integer such that $c_{j}=c_{k}$. By setting $x_{n}=0$ for $n>j+1, x_{0}=0$, the system $\left(\Lambda^{*}-c_{j} I\right) x=$ $\theta$ reduces to a homogeneous linear system of $j$ equations in 
$j+1$ unknowns, so that nontrivial solutions exist. Therefore $\Lambda-c_{j} I \in 3$.

$\Lambda-\alpha I$ is not one to one for $\alpha=0,1$ and so $\Lambda-\alpha I \in 3$. This step concludes the proof.

Lemma 12 (see [3, page 59]). T has a dense range if and only if $T^{*}$ is one to one.

Theorem 13. $\sigma_{r}\left(\Lambda, c_{0}\right)=\sigma_{p}\left(\Lambda^{*}, c_{0}^{*}\right) \backslash \sigma_{p}\left(\Lambda, c_{0}\right)$.

Proof. For $\alpha \in \sigma_{p}\left(\Lambda^{*}, c_{0}^{*}\right) \backslash \sigma_{p}\left(\Lambda, c_{0}\right)$, the operator $\Lambda-\alpha I$ is triangle, so has an inverse. But $\Lambda^{*}-\alpha I$ is not one to one by Theorem 10. Therefore by Lemma $12, \overline{R(\Lambda-\alpha I)} \neq c_{0}$, and this step concludes the proof.

Theorem 14. Let $\delta$ be defined as in Corollary 9 and $c_{n} \geq \delta$ for all sufficiently large $n$. Then,

$$
\sigma_{c}\left(\Lambda, c_{0}\right)=\left\{\alpha \in \mathbb{C}:\left|\alpha-\frac{1}{2-\delta}\right|=\frac{1-\delta}{2-\delta}, \alpha \neq 1, \frac{\delta}{2-\delta}\right\} .
$$

Proof. Fix $\alpha \neq 1, \delta /(2-\delta)$, and satisfying $|\alpha-1 /(2-\delta)|=$ $(1-\delta) /(2-\delta)$. Since the operator $\Lambda-\alpha I$ is triangle, it has an inverse. Consider the adjoint operator $\Lambda^{*}-\alpha I$. As in Theorem $11, x_{0}$ is arbitrary and

$$
x_{n}=\frac{\lambda_{n}-\lambda_{n-1}}{\lambda_{n-1}}\left(1-\frac{1}{\alpha}\right) x_{0} \prod_{j=k}^{n}\left[1+\left(1-\frac{1}{\alpha}\right) \frac{\lambda_{j}-\lambda_{j-1}}{\lambda_{j-1}}\right]
$$

for all positive $n$. From the hypothesis, there exists a positive integer $N$ such that $n \geq N$ implies $c_{n} \geq \delta$. This fact, together with the condition on $\alpha$, implies that $\mid 1+\left(1-\alpha^{-1}\right)\left(\lambda_{n}-\right.$ $\left.\lambda_{n-1}\right) / \lambda_{n-1} \mid \geq 1$ for $n \geq N$. Thus, $\left|x_{n}\right|=C\left(\lambda_{n}-\lambda_{n-1}\right) / \lambda_{n-1}$ for $n \geq N$, where $C$ is a positive constant independent of $n$. We can write

$$
\begin{aligned}
\frac{\lambda_{n}-\lambda_{n-1}}{\lambda_{n-1}} & =c_{n}\left(1+\frac{\lambda_{n}-\lambda_{n-1}}{\lambda_{n-1}}\right) \\
& \geq c_{n} .
\end{aligned}
$$

Therefore $\left(x_{n}\right) \in \ell_{1} \Leftrightarrow x_{0}=0$; that is, $\Lambda^{*}-\alpha I$ is one to one. From Lemma 12, the range of $\Lambda-\alpha I$ is dense in $c_{0}$. This completes the proof.

Lemma 15 (see [3, page 60]). T has a bounded inverse if and only if $T^{*}$ is onto.

Theorem 16. Let $\delta$ be defined as in Corollary 9 and less than 1. If $\alpha$ satisfies $|\alpha-1 /(2-\delta)|<(1-\delta) /(2-\delta)$ and $\alpha \notin S$, then $\alpha \in C_{1} \sigma\left(\Lambda, c_{0}\right)$.

Proof. First of all $\Lambda-\alpha I$ is a triangle; hence $1-1$. Therefore $\Lambda-\alpha I \in 1 \cup 2$. To verify that $\Lambda-\alpha I \in C_{1} \sigma\left(\Lambda, c_{0}\right)$ it is sufficient to show that $\Lambda^{*}-\alpha I$ is onto by Lemma 15 .

Suppose $y=\left(\Lambda^{*}-\alpha I\right) x$, where $x, y \in \ell_{1}$. Then, $x_{0}=$ $1 /(1-\alpha) y_{0}-\lambda_{0} /\left[\left(\lambda_{1}-\lambda_{0}\right)(1-\alpha)\right] y_{1}$ and

$$
\left(c_{n}-\alpha\right) x_{n}+\left(\lambda_{n}-\lambda_{n-1}\right) \sum_{k=n+1}^{\infty} \frac{x_{k}}{\lambda_{k}}=y_{n}, \quad n>0 .
$$

Choose $x_{1}=0$ and solve (51) for $x$ in terms of $y$ to get

$$
\begin{gathered}
\left(\lambda_{1}-\lambda_{0}\right) \sum_{k=2}^{\infty} \frac{x_{k}}{\lambda_{k}}=y_{1}, \\
\left(c_{n}-\alpha\right) x_{n}=y_{n}-\left(\lambda_{n}-\lambda_{n-1}\right) \sum_{k=n+1}^{\infty} \frac{x_{k}}{\lambda_{k}} .
\end{gathered}
$$

For example, substituting (52) into (53), with $n=2$, yields

$$
\left(c_{2}-\alpha\right) x_{2}=y_{2}-\left(\lambda_{2}-\lambda_{1}\right) \sum_{k=3}^{\infty} \frac{x_{k}}{\lambda_{k}}
$$

so that $x_{2}=\left(\lambda_{2}-\lambda_{1}\right) /\left[\alpha\left(\lambda_{1}-\lambda_{0}\right)\right] y_{1}-(1 / \alpha) y_{2}$. For $n=3$,

$$
x_{3}=-\left(\frac{\lambda_{3}-\lambda_{2}}{\lambda_{1}-\lambda_{0}}\right)\left(c_{2}-\alpha\right) \frac{1}{\alpha^{2}} y_{1}+\left(\frac{\lambda_{3}-\lambda_{2}}{\lambda_{2}}\right) \frac{1}{\alpha^{2}} y_{2}-\frac{1}{\alpha} y_{3} \text {. }
$$

Continuing this process, the entries of the matrix $B=$ $\left(b_{n k}\right)$ such that $B y=x$ are calculated as

$$
\begin{gathered}
b_{00}=\frac{1}{1-\alpha}, \quad b_{01}=-\frac{\lambda_{0}}{\left(\lambda_{1}-\lambda_{0}\right)(1-\alpha)} \\
b_{21}=\frac{\lambda_{2}-\lambda_{1}}{\left(\lambda_{1}-\lambda_{0}\right) \alpha}, \quad b_{n n}=-\frac{1}{\alpha}, \quad n>1, \\
b_{n, n-1}=\frac{\lambda_{n}-\lambda_{n-1}}{\lambda_{n-1} \alpha^{2}}, \quad n>2 \\
b_{n 1}=\frac{\lambda_{n}-\lambda_{n-1}}{\left(\lambda_{1}-\lambda_{0}\right) \alpha} \prod_{j=2}^{n-1}\left(1-\frac{c_{j}}{\alpha}\right), \quad n>2, \\
b_{n k}=\frac{\lambda_{n}-\lambda_{n-1}}{\lambda_{k} \alpha^{2}} \prod_{j=k+1}^{n-1}\left(1-\frac{c_{j}}{\alpha}\right), \quad 1<k<n-1,
\end{gathered}
$$

and $b_{n k}=0$ otherwise.

To show that $B \in B\left(\ell_{1}\right)$, it is sufficient to establish that $\sum_{n=0}^{\infty}\left|b_{n k}\right|$ is finite independent of $k . \sum_{n=0}^{\infty}\left|b_{n 0}\right|=1 /|1-\alpha|$. We may write $1-\left(c_{j} / \alpha\right)=\left(\lambda_{j-1} / \lambda_{j}\right)\left[1+\left(1-\alpha^{-1}\right)\left(\lambda_{j}-\lambda_{j-1}\right) / \lambda_{j-1}\right]$. Also, $\sup _{n \in \mathbb{N}}\left|\left(\lambda_{n}-\lambda_{n-1}\right) / \lambda_{n-1}\right| \leq M<\infty$. Therefore,

$$
\sum_{n=0}^{\infty}\left|b_{n 1}\right| \leq \frac{1}{|\alpha|}\left[M+M \sum_{n=3}^{\infty} \prod_{j=2}^{n-1}\left|1+\left(1-\frac{1}{\alpha}\right) \frac{\lambda_{j}-\lambda_{j-1}}{\lambda_{j-1}}\right|\right]
$$

and, for $k>1$,

$$
\begin{aligned}
\sum_{n=0}^{\infty}\left|b_{n k}\right| \leq & \frac{1}{|\alpha|}+\frac{M}{|\alpha|^{2}} \\
& +\frac{M}{|\alpha|^{2}} \sum_{n=k+2}^{\infty} \prod_{j=k+1}^{n-1}\left|1+\left(1-\frac{1}{\alpha}\right) \frac{\lambda_{j}-\lambda_{j-1}}{\lambda_{j-1}}\right| .
\end{aligned}
$$

Since $k>1$, the series in inequality (24) is absolutely convergent from Theorem 7. Therefore, $\|B\|_{\left(\ell_{1}: \ell_{1}\right)}$ is finite.

Because of $(\Lambda-\alpha I)^{-1}$ is bounded, it is continuous, and $\alpha \in C_{1} \sigma\left(\Lambda, c_{0}\right)$. This completes the proof. 
Theorem 17. Let $\delta$ be defined as in Corollary 9 and $\delta<1$. If $\alpha=\delta$ or $\alpha=c_{n}$ for all $n \in \mathbb{N}$ and $\delta /(2-\delta)<\alpha<1$, then $\alpha \in C_{1} \sigma\left(\Lambda, c_{0}\right)$.

Proof. First assume that $\Lambda$ has distinct diagonal entries and fix $j \geq 1$. Then the system $\left(\Lambda-c_{j} I\right) x=\theta$ implies that $x_{n}=0$ for $n=0,1, \ldots, j-1$, and for $n \geq j$

$$
\left(c_{j}-c_{n}\right) x_{n}-\sum_{k=0}^{n-1} \lambda_{n k} x_{k}=0 .
$$

The system (59) yields the following recursion relation:

$$
x_{n+1}=\frac{\lambda_{n} c_{j} x_{n}}{\lambda_{n+1}\left(c_{j}-c_{n+1}\right)}
$$

which can be solved for $x_{n}$ to yield

$$
\begin{aligned}
x_{j+m} & =\frac{\lambda_{j} x_{j} c_{j}^{m}}{\lambda_{j+m} \prod_{i=1}^{m}\left(c_{j}-c_{j+i}\right)}=x_{j} \prod_{i=1}^{m} \frac{\lambda_{j+i-1}}{\lambda_{j+i}\left(1-c_{j+i} / c_{j}\right)} \\
& =x_{j}\left\{\prod_{i=1}^{m} \frac{\lambda_{j+i}}{\lambda_{j+i-1}}\left[1-\frac{\lambda_{j}\left(\lambda_{j+i}-\lambda_{j+i-1}\right)}{\lambda_{j+i}\left(\lambda_{j}-\lambda_{j-1}\right)}\right]\right\}^{-1} \\
& =x_{j}\left\{\prod_{i=1}^{m}\left[\frac{\lambda_{j+i}}{\lambda_{j+i-1}}-\frac{\lambda_{j}\left(\lambda_{j+i}-\lambda_{j+i-1}\right)}{\lambda_{j+i-1}\left(\lambda_{j}-\lambda_{j-1}\right)}\right]\right\}^{-1} \\
& =x_{j}\left\{\prod_{i=1}^{m}\left[\frac{\lambda_{j+i}}{\lambda_{j+i-1}}-\frac{\lambda_{j+i}-\lambda_{j+i-1}}{\lambda_{j+i-1}}\right]\right\}^{-1} \\
& =x_{j}\left\{\prod_{i=1}^{m}\left[1+\left(1-\frac{1}{c_{j}}\right) \frac{\lambda_{j+i}-\lambda_{j+i-1}}{\lambda_{j+i-1}}\right]\right\}^{-1} .
\end{aligned}
$$

Since $0<c_{j}<1$, the argument of Theorem 7 applies and (24) is true. Therefore $x \in c_{0}$ implies $x=\theta$ and $\Lambda-c_{j} I$ is $1-1$, so that $\Lambda-c_{j} I \in 1 \cup 2$. onto.

Clearly $\Lambda-c_{j} I \in C$. It remains to show that $\Lambda^{*}-c_{j} I$ is

Suppose that $\left(\Lambda^{*}-c_{j} I\right) x=y$, where $x, y \in \ell_{1}$. By choosing $x_{j+1}=0$ we can solve for $x_{0}, x_{1}, \ldots, x_{j}$ in terms of $y_{0}, y_{1}, \ldots, y_{j+1}$. As in Theorem 16 , the remaining equations can be written in the form $x=B y$, where the nonzero entries of $B=\left(b_{n k}\right)$ are as follows

$$
\begin{gathered}
b_{j+m, j+m}=-\frac{1}{c_{j}} ; \\
b_{j+2, j+1}=\frac{\lambda_{j+2}-\lambda_{j+1}}{c_{j}\left(\lambda_{j+1}-\lambda_{j}\right)} ; \\
b_{j+m, j+m-1}=\frac{\lambda_{j+m}-\lambda_{j+m-1}}{c_{j}^{2} \lambda_{j+m-1}}, \quad m>2 ;
\end{gathered}
$$

$$
\begin{gathered}
b_{j+m, j+k}=\frac{\lambda_{j+m}-\lambda_{j+m-1}}{c_{j}^{2} \lambda_{j+k}} \prod_{i=j+k+1}^{j+m-1}\left(1-\frac{c_{i}}{c_{j}}\right), \\
1<k<m-1, m>3 ; \\
b_{j+m, j+1}=\frac{\lambda_{j+m}-\lambda_{j+m-1}}{c_{j}\left(\lambda_{j+1}-\lambda_{j}\right)} \prod_{i=j+2}^{j+m-1}\left(1-\frac{c_{i}}{c_{j}}\right), \quad m>2 .
\end{gathered}
$$

From (62), we have

$$
\begin{aligned}
\sum_{n=j+1}^{\infty}\left|b_{n, j+1}\right|= & \frac{\lambda_{j+2}-\lambda_{j+1}}{c_{j}\left(\lambda_{j+1}-\lambda_{j}\right)}+\frac{1}{c_{j}\left(\lambda_{j+1}-\lambda_{j}\right)} \\
& \times \sum_{n=j+3}^{\infty}\left(\lambda_{n}-\lambda_{n-1}\right) \prod_{i=j+2}^{n-1}\left|1-\frac{c_{i}}{c_{j}}\right| .
\end{aligned}
$$

For $m>1$,

$$
\begin{aligned}
\sum_{n=m+j}^{\infty}\left|b_{n, m+j}\right|= & \frac{1}{c_{j}}+\frac{\lambda_{j+m+1}-\lambda_{j+m}}{c_{j}^{2} \lambda_{j+m}} \\
& +\frac{1}{c_{j}^{2}} \sum_{n=j+m+2}^{\infty} \frac{\lambda_{n}-\lambda_{n-1}}{\lambda_{j+m}} \prod_{i=j+m+1}^{n-1}\left|1-\frac{c_{i}}{c_{j}}\right| .
\end{aligned}
$$

Using $1-\left(c_{j} / \alpha\right)=\left(\lambda_{j-1} / \lambda_{j}\right)\left[1+\left(1-\alpha^{-1}\right)\left(\lambda_{j}-\lambda_{j-1}\right) / \lambda_{j-1}\right]$, one can convert (63) and (64) the similar expressions to (57) and (58), and therefore $\|B\|_{\left(\ell_{1}: \ell_{1}\right)}$ is finite.

Suppose that $\Lambda$ does not have distinct diagonal entries. The restriction on $\alpha$ guarantees that no zero diagonal entries are being considered. Let $c_{j} \neq 0$ be any diagonal entry which occurs more than once, and let $k, r$ denote, respectively, the smallest and largest integers for which $c_{j}=c_{k}=c_{r}$. From (61) it follows that $x_{n}=0$ for $n \geq r$. Also, $x_{n}=0$ for $0 \leq n<k$. Therefore the system $\left(\Lambda-c_{j} I\right) x=\theta$ becomes

$$
\left(c_{j}-c_{n}\right) x_{n}-\sum_{i=j}^{n-1} \lambda_{n i} x_{i}=0, \quad k<n \leq r .
$$

Case 1. Let $r=k+1$. Then (65) reduces to the single equation

$$
\left(c_{j}-c_{k+1}\right) x_{j+1}-\left(\frac{\lambda_{k}-\lambda_{k-1}}{\lambda_{k+1}}\right) x_{k}=0
$$

which implies that $x_{k}=0$, since $c_{j}=c_{r}=c_{k+1}$ and $c_{j} \neq 0$. Therefore $x=\theta$.

Case 2. Let $r>k+1$. From (65) one can obtain the recursion formula $x_{n}=\lambda_{n+1}\left(c_{j}-c_{n+1}\right) x_{n+1} /\left(\lambda_{n} c_{j}\right)$ with $k<n<r$. Since $x_{r}=0$ it then follows that $x_{n}=0$ for $k<n<r$. Using (65) with $n=k+1$ yields $x_{k}=0$ and so again $x=\theta$.

To show that $\Lambda^{*}-c_{j} I$ is onto, suppose $\left(\Lambda^{*}-c_{j} I\right) x=y$, where $x, y \in \ell_{1}$. By choosing $x_{j+1}=0$ one can solve for $x_{0}, x_{1}, \ldots, x_{j}$ in terms of $y_{0}, y_{1}, \ldots, y_{j+1}$. As in Theorem 16, the remaining equations can be written in the form $x=B y$, 
where the nonzero entries of $B$ are as in (62) with the other entries of $B$ clearly zero.

Since $k \leq j \leq r$, there are two cases to consider.

Case 1. If $j=r$, then the proof proceeds exactly as in the argument following (62).

Case 2. If $j<r$, then from (62), $b_{j+m, j+k}=b_{j+m, j+1}=0$ at least for $m \geq r-j+2$. If there are other values of $n$ with $j<n<r$ for which $c_{n}-c_{j}$, then additional entries of $B$ will be zero. These zero entries do not affect the validity of the argument showing that (63) converges.

If $\delta=0$, then 0 does not lie inside the disc, and so it is not considered in this theorem.

Let $\alpha=\delta>0$. If $\lambda_{n n} \leq \delta$ for each $n \geq 1$, all $i$ sufficiently large, then the argument of Theorem 16 applies and $\Lambda-\delta I \epsilon$ $C_{1}$. If $\lambda_{n n}=\delta$ for some $n$, then the proof of Theorem 17 applies with $c_{j}$ replaced by $\delta$ and again, $\Lambda-\delta I \in C_{1}$.

Therefore, in all cases, $\Lambda-c_{j} I \in 1 \cup 2$.

Theorem 18. If $\alpha \in \sigma_{p}\left(\Lambda, c_{0}\right), \alpha \in C_{3} \sigma\left(\Lambda, c_{0}\right)$.

Proof. For $\alpha \in \sigma_{p}\left(\Lambda, c_{0}\right), \Lambda-\alpha I \in 3$ and $\Lambda^{*}-\alpha I$ is not one to one. Therefore $\overline{R(\Lambda-\alpha I)} \neq c_{0}$ by Lemma 12. This concludes the proof.

Theorem 19. The statement $A_{3} \sigma\left(\Lambda, c_{0}\right)=C_{2} \sigma\left(\Lambda, c_{0}\right)=\emptyset$ holds.

Proof. Let $\delta$ be defined as in Corollary 9 and $c_{n} \geq \delta$ for all sufficiently large $n$, then $A_{3} \sigma\left(\Lambda, c_{0}\right)=\emptyset$ and $C_{2} \sigma\left(\Lambda, c_{0}\right)=\emptyset$ follow from Corollary 9, Theorems 14, and 16-18.

Define the set $E$ by

$$
E=\overline{\left\{c_{j}: c_{j} \leq \frac{\eta}{2-\eta}\right\}}
$$

where $\eta$ is as in Theorem 7 .

We will consider $\delta=\eta$, that is, for which the main diagonal entries converge, where $\delta$ as in Corollary 9 .

Theorem 20. The following results hold:

(a) $\sigma_{a p}\left(\Lambda, c_{0}\right)=\left\{\alpha:\left|\alpha-(2-\delta)^{-1}\right|=(1-\delta) /(2-\delta)\right\} \cup E$,

(b) $\sigma_{\delta}\left(\Lambda, c_{0}\right)=\sigma\left(\Lambda, c_{0}\right)$,

(c) $\sigma_{c o}\left(\Lambda, c_{0}\right)=\left\{\alpha \in \mathbb{C}:\left|\alpha-(2-\delta)^{-1}\right|<(1-\delta) /(2-\delta)\right\} \cup S$.

Proof. (a) Since the relation

$$
\begin{aligned}
C_{1} \sigma\left(\Lambda, c_{0}\right)= & \left\{\left\{\alpha:\left|\alpha-\frac{1}{2-\delta}\right|<\frac{1-\delta}{2-\delta}\right\} \backslash S\right\} \\
& \bigcup\left\{\alpha=\lambda_{n n}: \frac{\delta}{2-\delta}<\alpha<1\right\}
\end{aligned}
$$

holds by Theorems 16 and 17 and from Table $1, \sigma_{\text {ap }}\left(\Lambda, c_{0}\right)=$ $\sigma\left(\Lambda, c_{0}\right) \backslash C_{1} \sigma\left(\Lambda, c_{0}\right)$. Therefore, we have $\sigma_{\text {ap }}\left(\Lambda, c_{0}\right)=\{\alpha: \mid \alpha-$ $\left.(2-\delta)^{-1} \mid=(1-\delta) /(2-\delta)\right\} \cup E$. (b) Since $\sigma_{\delta}\left(\Lambda, c_{0}\right)=\sigma\left(\Lambda, c_{0}\right) \backslash A_{3} \sigma\left(\Lambda, c_{0}\right)$ from Table 1 and $A_{3} \sigma\left(\Lambda, c_{0}\right)=\emptyset$ by Theorem 19 , we have $\sigma_{\delta}\left(\Lambda, c_{0}\right)=\sigma\left(\Lambda, c_{0}\right)$.

(c) Since the equality $\sigma_{\text {co }}\left(\Lambda, c_{0}\right)=C_{1} \sigma\left(\Lambda, c_{0}\right) \cup C_{2} \sigma\left(\Lambda, c_{0}\right) \cup$ $C_{3} \sigma\left(\Lambda, c_{0}\right)$ holds from Table 1 , we have $\sigma_{\text {co }}\left(\Lambda, c_{0}\right)=\{\alpha \in \mathbb{C}$ : $\left.\left|\alpha-(2-\delta)^{-1}\right|<(1-\delta) /(2-\delta)\right\} \cup S$ by Theorems 16-19.

The next corollary can be obtained from Proposition 1.

Corollary 21. The following results hold:

(a) $\sigma_{a p}\left(\Lambda^{*}, \ell_{1}\right)=\sigma\left(\Lambda, c_{0}\right)$,

(b) $\sigma_{\delta}\left(\Lambda^{*}, \ell_{1}\right)=\left\{\alpha:\left|\alpha-(2-\delta)^{-1}\right|=(1-\delta)(2-\delta)\right\} \cup E$,

(c) $\sigma_{p}\left(\Lambda^{*}, \ell_{1}\right)=\left\{\alpha \in \mathbb{C}:\left|\alpha-(2-\delta)^{-1}\right|<(1-\delta) /(2-\right.$ $\delta)\} \cup S$.

\section{The Fine Spectrum of the Operator $\Lambda$ on the Sequence Space $c$}

In this section, we investigate the fine spectrum of the operator $\Lambda$ over the sequence space $c$.

Theorem 22. $\sigma(\Lambda, c) \subseteq\{\alpha \in \mathbb{C}:|2 \alpha-1| \leq 1\}$.

Proof. This is obtained in a similar way to that used in the proof of Theorem 6 .

Theorem 23. Suppose that $\mu, \eta$ and $S$ be defined as in Theorem 7. Then,

$$
\begin{aligned}
\{\alpha \in & \left.\mathbb{C}:\left|\alpha-\frac{1}{2-\mu}\right| \leq \frac{1-\mu}{2-\mu}\right\} \cup S \\
& \subseteq \sigma(\Lambda, c) \\
& \subseteq\left\{\alpha \in \mathbb{C}:\left|\alpha-\frac{1}{2-\eta}\right| \leq \frac{1-\eta}{2-\eta}\right\} \cup S .
\end{aligned}
$$

Proof. This is similar to the proof of Theorems 7 and 8. To avoid the repetition of the similar statements, we omit the detail.

Corollary 24. Let $\delta$ be defined as in Corollary 9. Then,

$$
\sigma(\Lambda, c)=\left\{\alpha \in \mathbb{C}:\left|\alpha-\frac{1}{2-\delta}\right| \leq \frac{1-\delta}{2-\delta}\right\} \cup S .
$$

If $T: c \rightarrow c$ is a bounded linear operator with the matrix $A$, then $T^{*}: c^{*} \rightarrow c^{*}$ acting on $\mathbb{C} \oplus \ell_{1}$ has a matrix representation of the form

$$
\left[\begin{array}{cc}
\chi & 0 \\
b & A^{t}
\end{array}\right]
$$

where $\chi$ is the limit of the sequence of row sums of $A$ minus the sum of the limit of the columns of $A$ and $b$ is the column vector whose $k$ th entry is the limit of the $k$ th column of $A$ for each $k \in \mathbb{N}$. For $\Lambda: c \rightarrow c$, the matrix $\Lambda^{*} \in B\left(\ell_{1}\right)$ is of the form

$$
\Lambda^{*}=\left[\begin{array}{cc}
1 & 0 \\
0 & \Lambda^{t}
\end{array}\right]
$$


Theorem 25. Let $\delta$ be defined as in Corollary 9. Then,

$$
\sigma_{p}\left(\Lambda^{*}, c^{*}\right)=\left\{\alpha \in \mathbb{C}:\left|\alpha-\frac{1}{2-\delta}\right|<\frac{1-\delta}{2-\delta}\right\} \cup S .
$$

Proof. Suppose that $\Lambda^{*} x=\alpha x$ for $x \neq \theta$ in $c^{*} \cong \ell_{1}$. Then, by solving the system of linear equations

$$
\begin{gathered}
(1-\alpha) x_{0}=0 \\
x_{2}=\frac{\lambda_{1}-\lambda_{0}}{\lambda_{0}}\left(1-\frac{1}{\alpha}\right) x_{1}, \\
x_{3}=\frac{\lambda_{2}-\lambda_{1}}{\lambda_{0}}\left(1-\frac{c_{1}}{\alpha}\right)\left(1-\frac{1}{\alpha}\right) x_{1} \\
\vdots \\
x_{n}=\frac{\lambda_{n-1}-\lambda_{n-2}}{\lambda_{0}}\left(1-\frac{1}{\alpha}\right) x_{1} \prod_{j=1}^{n-1}\left(1-\frac{c_{j}}{\alpha}\right)
\end{gathered}
$$

we get by assumption $(1-\alpha) x_{0}=0$ with $\alpha=1$ that $x=$ $\left(x_{0}, x_{1}, 0,0, \ldots\right) \in c$. If $\alpha \neq 1$, we have $x_{0}=0$ and $\left(x_{n}\right) \in \ell_{1}$ if and only if $\left|1+\left(1-\alpha^{-1}\right)\left(\lambda_{j}-\lambda_{j-1}\right) / \lambda_{j-1}\right|<1$, by Theorem 11 . This completes the proof.

Theorem 26. Let $\delta$ be defined as in Corollary 9. Then,

$$
\sigma_{p}(\Lambda, c)=\left\{\alpha=c_{n} \in \mathbb{C}: 0 \leq \alpha \leq \frac{\delta}{2-\delta}\right\} \cup\{1\} .
$$

Proof. The proof is identical to the proof of Theorem 11.

Theorem 27. $\sigma_{r}(\Lambda, c)=\sigma_{p}\left(\Lambda^{*}, c^{*}\right) \backslash \sigma_{p}(\Lambda, c)$.

Proof. For $\alpha \in \sigma_{p}\left(\Lambda^{*}, c^{*}\right) \backslash \sigma_{p}(\Lambda, c)$, the operator $\Lambda-\alpha I$ is triangle, so has an inverse. But $\Lambda^{*}-\alpha I$ is not one to one by Theorem 26. Therefore by Lemma $12, \overline{R(\Lambda-\alpha I)} \neq c$ and this concludes the proof.

Since Theorems 28-31 can be proved in a similar way to that used in the proof of Theorems 14 and 16-18; respectively, to avoid the repetition of the similar statements we omit the detailed proof and give them without proof.

Theorem 28. Let $\delta$ be defined as in Corollary 9 and $c_{n} \geq \delta$ for all sufficiently large $n$. Then,

$$
\sigma_{c}(\Lambda, c)=\left\{\alpha \in \mathbb{C}:\left|\alpha-\frac{1}{2-\delta}\right|=\frac{1-\delta}{2-\delta}, \alpha \neq 1, \frac{\delta}{2-\delta}\right\} .
$$

Theorem 29. Let $\delta$ be defined as in Corollary 9 and less than 1. If $\alpha$ satisfies $|\alpha-1 /(2-\delta)|<(1-\delta) /(2-\delta)$ and $\alpha \notin S$, then $\alpha \in C_{1} \sigma(\Lambda, c)$.

Theorem 30. Let $\delta$ be defined as in Corollary 9 and $\delta<1$. If $\alpha=\delta$ or $\alpha=c_{n}$ for all $n \in \mathbb{N}$ and $\delta /(2-\delta)<\alpha<1$, then $\alpha \in C_{1} \sigma(\Lambda, c)$.
Theorem 31. If $\alpha \in \sigma_{p}(\Lambda, c), \alpha \in C_{3} \sigma(\Lambda, c)$.

Theorem 32. The following statement holds: $A_{3} \sigma(\Lambda, c)=$ $C_{2} \sigma(\Lambda, c)=\emptyset$.

Proof. Let $\delta$ be defined as in Corollary 9 and $c_{n} \geq \delta$ for all sufficiently large $n$, then $A_{3} \sigma(\Lambda, c)=\emptyset$ and $C_{2} \sigma(\Lambda, c)=\emptyset$ follow from Corollary 24 and Theorems 28-31.

Theorem 33. The following results hold:
(a) $\sigma_{a p}(\Lambda, c)=\left\{\alpha:\left|\alpha-(2-\delta)^{-1}\right|=(1-\delta) /(2-\delta)\right\} \cup E$,
(b) $\sigma_{\delta}(\Lambda, c)=\sigma(\Lambda, c)$,
(c) $\sigma_{c o}(\Lambda, c)=\left\{\alpha \in \mathbb{C}:\left|\alpha-(2-\delta)^{-1}\right|<(1-\delta) /(2-\delta)\right\} \cup S$.

Proof. (a) Since the relation $C_{1} \sigma(\Lambda, c)=\left\{\left\{\alpha:\left|\alpha-(2-\delta)^{-1}\right|<\right.\right.$ $(1-\delta) /(2-\delta)\} \backslash S\} \bigcup\left\{\alpha=\lambda_{n n}: \delta /(2-\delta)<\alpha<1\right\}$ holds by Theorems 29 and 30 and from Table $1, \sigma_{\text {ap }}(\Lambda, c)=\sigma(\Lambda, c) \backslash$ $C_{1} \sigma(\Lambda, c)$. Therefore, we have $\sigma_{\text {ap }}(\Lambda, c)=\left\{\alpha:\left|\alpha-(2-\delta)^{-1}\right|=\right.$ $(1-\delta) /(2-\delta)\} \cup E$.

(b) Since $\sigma_{\delta}(\Lambda, c)=\sigma(\Lambda, c) \backslash A_{3} \sigma(\Lambda, c)$ from Table 1 and $A_{3} \sigma(\Lambda, c)=\emptyset$ by Theorem 32 , we have $\sigma_{\delta}(\Lambda, c)=\sigma(\Lambda, c)$.

(c) Since the equality $\sigma_{\text {co }}(\Lambda, c)=C_{1} \sigma(\Lambda, c) \cup C_{2} \sigma(\Lambda, c) \cup$ $C_{3} \sigma(\Lambda, c)$ holds from Table 1 , we have $\sigma_{\text {co }}(\Lambda, c)=\{\alpha \in \mathbb{C}$ : $\left.\left|\alpha-(2-\delta)^{-1}\right|<(1-\delta) /(2-\delta)\right\} \cup S$ by Theorems 29-32.

The next corollary can be obtained from Proposition 1.

Corollary 34. The following results hold:

(a) $\sigma_{a p}\left(\Lambda^{*}, \ell_{1}\right)=\sigma(\Lambda, c)$,

(b) $\sigma_{\delta}\left(\Lambda^{*}, \ell_{1}\right)=\left\{\alpha:\left|\alpha-(2-\delta)^{-1}\right|=(1-\delta) /(2-\delta)\right\} \cup E$,

(c) $\sigma_{p}\left(\Lambda^{*}, \ell_{1}\right)=\left\{\alpha \in \mathbb{C}:\left|\alpha-(2-\delta)^{-1}\right|<(1-\delta) /(2-\right.$ $\delta)\} \cup S$.

Let $A$ be an infinite matrix and let the set $c_{A}$ denote the convergence domain of that matrix $A$, a theorem which proves that $c_{A}=c$ is called a Mercerian theorem, after Mercer, who proved a significant theorem of this type [28, page 186].

Now, we may give our final theorem.

Theorem 35. Suppose that $|\alpha+1|>|\alpha-1|$. Then the convergence field of $A=\alpha I+(1-\alpha) \Lambda$ is $c$.

Proof. By Theorem 22, $\Lambda-[\alpha /(\alpha-1)] I$ has an inverse in $B(c)$. That is to say that

$$
A^{-1}=\frac{1}{1-\alpha}\left(\Lambda-\frac{\alpha}{\alpha-1} I\right)^{-1} \in B(c) .
$$

Since $A$ is a triangle and is in $B(c), A^{-1}$ is also conservative which implies that $c_{A}=c$ [22, page 12].

\section{Conclusion}

Although the matrix $\Lambda$ is used for obtaining some new sequence spaces by its domain from the classical sequence spaces, it is not considered for determining the spectrum or fine spectrum acting as a linear operator on any of the 
classical sequence spaces $c_{0}, c$, or $\ell_{p}$. Following Altay and Başar [12] and Karakaya and Altun [26], we determine the fine spectrum with respect to Goldberg's classification of the operator defined by the triangle matrix $\Lambda$ over the sequence spaces $c_{0}$ and $c$ which reduces to a new regular triangle matrix depending on choosing the strictly increasing sequence $\lambda=$ $\left(\lambda_{k}\right)$ of positive real numbers tending to infinity. Additionally, we give the approximate point spectrum, the defect spectrum, and the compression spectrum of the matrix operator $\Lambda$ over the spaces $c_{0}$ and $c$. Since the present paper is devoted to the fine spectrum of the operator defined by the lambda matrix over the sequence spaces $c_{0}$ and $c$ with new subdivision of spectrum, this makes it significant. We should note that the main results of the present paper are given as an extended abstract without proof by Yeşilkayagil and Başar [29]. by

The generalized weighted means $G(u, v)=\left(g_{n k}\right)$ is defined

$$
g_{n k}= \begin{cases}u_{n} v_{k}, & 0 \leq k \leq n, \\ 0, & k>n,\end{cases}
$$

for all $k, n \in \mathbb{N}$, where $u_{n}$ depends only on $n$ and $v_{k}$ only on $k$ such that $u_{n}, v_{k} \neq 0$. It is immediate that in the case $u_{n}=1 / \lambda_{n}$ and $v_{k}=\lambda_{k}-\lambda_{k-1}$, the generalized weighted means $G(u, v)$ corresponds to the matrix $\Lambda$. Although the Riesz means $R^{q}$, the generalized weighted means $G(u, v)$, and the matrix $\Lambda$ were used for different purposes, their fine spectrum over the classical sequence spaces was not studied. As a beginning, the present work has an advantage.

Finally, we record from now on that our next paper will be devoted to the investigation of the fine spectrum of the matrix operator $\Lambda$ on the spaces $\ell_{p}$ and $b v_{p}$ in the cases $0<p<1$ and $1 \leq p<\infty$, where $b v_{p}$ denotes the space of all sequences whose $\Delta$-transforms are in the space $\ell_{p}$ and was studied in the case $0<p<1$ by Altay and Başar [30] and in the case $1 \leq p \leq \infty$ by Başar and Altay [31].

\section{Acknowledgments}

The authors would like to express their pleasure to Professor Bilâl Altay, Department of Mathematical Education, Faculty of Education, İnönü University, Malatya, Turkey, for many helpful suggestions and interesting comments on the main results of the earlier version of the paper. Additionally, the authors are very grateful to the referee for making some useful remarks which improved the presentation of the paper. The main results of this paper has been presented in part at the conference First International Conference on Analysis and Applied Mathematics (ICAAM 2012) to be held on October 18-21, 2012, in Gümüşhane, Turkey, at the University of Gümüşhane and published in the conference proceedings with AIP, as an extended abstract.

\section{References}

[1] E. Kreyszig, Introductory Functional Analysis with Applications, John Wiley \& Sons, New York, NY, USA, 1978.
[2] J. Appell, E. De Pascale, and A. Vignoli, Nonlinear Spectral Theory, vol. 10 of de Gruyter Series in Nonlinear Analysis and Applications, Walter de Gruyter, Berlin, Germany, 2004.

[3] S. Goldberg, Unbounded Linear Operators, Dover Publications Inc., New York, NY, USA, 1985.

[4] R. B. Wenger, "The fine spectra of the Hölder summability operators," Indian Journal of Pure and Applied Mathematics, vol. 6, no. 6, pp. 695-712, 1975.

[5] B. E. Rhoades, "The fine spectra for weighted mean operators," Pacific Journal of Mathematics, vol. 104, no. 1, pp. 219-230, 1983.

[6] J. B. Reade, "On the spectrum of the Cesàro operator," The Bulletin of the London Mathematical Society, vol. 17, no. 3, pp. 263-267, 1985.

[7] A. M. Akhmedov and F. Başar, "On the fine spectrum of the Cesàro operator in $c_{0}$," Mathematical Journal of Ibaraki University, vol. 36, pp. 25-32, 2004.

[8] J. T. Okutoyi, "On the spectrum of $C_{1}$ as an operator on bv," Communications, Faculty of Science, University of Ankara. Series A1, vol. 41, no. 1-2, pp. 197-207, 1992.

[9] M. Yildırım, "On the spectrum and fine spectrum of the compact Rhaly operators," Indian Journal of Pure and Applied Mathematics, vol. 27, no. 8, pp. 779-784, 1996.

[10] C. Coşkun, "The spectra and fine spectra for $p$-Cesàro operators," Turkish Journal of Mathematics, vol. 21, no. 2, pp. 207-212, 1997.

[11] B. de Malafosse, "Properties of some sets of sequences and application to the spaces of bounded difference sequences of order $\mu$," Hokkaido Mathematical Journal, vol. 31, no. 2, pp. 283299, 2002.

[12] B. Altay and F. Başar, "On the fine spectrum of the difference operator $\Delta$ on $c_{0}$ and c," Information Sciences, vol. 168, no. 1-4, pp. 217-224, 2004.

[13] H. Bilgiç and H. Furkan, "On the fine spectrum of the operator $B(r, s, t)$ over the sequence spaces $l_{1}$ and $b v$," Mathematical and Computer Modelling, vol. 45, no. 7-8, pp. 883-891, 2007.

[14] H. Furkan, H. Bilgiç, and F. Bașar, "On the fine spectrum of the operator $B(r, s, t)$ over the sequence spaces $\ell_{p}$ and $b v_{p},(1<p<$ $\infty)$," Computers \& Mathematics with Applications, vol. 60, no. 7, pp. 2141-2152, 2010.

[15] A. M. Akhmedov and S. R. El-Shabrawy, "On the fine spectrum of the operator $\Delta_{a, b}$ over the sequence space $c$," Computers \& Mathematics with Applications, vol. 61, no. 10, pp. 2994-3002, 2011.

[16] P. D. Srivastava and S. Kumar, "Fine spectrum of the generalized difference operator $\Delta_{v}$ on sequence space $l_{1}$," Thai Journal of Mathematics, vol. 8, no. 2, pp. 221-233, 2010.

[17] B. L. Panigrahi and P. D. Srivastava, "Spectrum and fine spectrum of generalized second order difference operator $\Delta_{u v}^{2}$ on sequence space $c_{0}$," Thai Journal of Mathematics, vol. 9, no. 1, pp. 57-74, 2011.

[18] P. D. Srivastava and S. Kumar, "Fine spectrum of the generalized difference operator $\Delta_{u v}$ on sequence space $l_{1}$," Applied Mathematics and Computation, vol. 218, no. 11, pp. 6407-6414, 2012.

[19] A. Karaisa and F. Başar, "Fine spectra of upper triangular tripleband matrix over the sequence space $\ell_{p},(0<p<\infty)$," Abstract and Applied Analysis. In press.

[20] M. Mursaleen and A. K. Noman, "On the spaces of $\lambda$-convergent and bounded sequences," Thai Journal of Mathematics, vol. 8, no. 2, pp. 311-329, 2010.

[21] F. Móricz, "On $\Lambda$-strong convergence of numerical sequences and Fourier series," Acta Mathematica Hungarica, vol. 54, no. 3-4, pp. 319-327, 1989. 
[22] A. Wilansky, Summability through Functional Analysis, vol. 85 of North-Holland Mathematics Studies, North-Holland Publishing, Amsterdam, The Netherlands, 1984.

[23] M. González, "The fine spectrum of the Cesàro operator in $\ell_{p}(1<p<\infty)$," Archiv der Mathematik, vol. 44, no. 4, pp. 355-358, 1985.

[24] B. Altay and M. Karakuş, "On the spectrum and the fine spectrum of the Zweier matrix as an operator on some sequence spaces," Thai Journal of Mathematics, vol. 3, no. 2, pp. 153-162, 2005.

[25] M. Altun, "On the fine spectra of triangular Toeplitz operators," Applied Mathematics and Computation, vol. 217, no. 20, pp. 8044-8051, 2011.

[26] V. Karakaya and M. Altun, "Fine spectra of upper triangular double-band matrices," Journal of Computational and Applied Mathematics, vol. 234, no. 5, pp. 1387-1394, 2010.

[27] F. P. Cass and B. E. Rhoades, "Mercerian theorems via spectral theory," Pacific Journal of Mathematics, vol. 73, no. 1, pp. 63-71, 1977.

[28] I. J. Maddox, Elements of Functional Analysis, Cambridge University Press, London, UK, 1970.

[29] M. Yeşilkayagil and F. Başar, "On the fine spectrum of the operator defined by a lambda matrix over the sequence space $c_{0}$ and $c$," in Proceedings of the 1st International Conference on Analysis and Applied Mathematics (ICAAM '12), vol. 1470 of AIP Conference Proceedings, pp. 199-202, October 2012.

[30] B. Altay and F. Başar, "The fine spectrum and the matrix domain of the difference operator $\Delta$ on the sequence space $\ell_{p},(0<p<$ 1)," Communications in Mathematical Analysis, vol. 2, no. 2, pp. $1-11,2007$.

[31] F. Başar and B. Altay, "On the space of sequences of $p$ bounded variation and related matrix mappings," Ukrainian Mathematical Journal, vol. 55, no. 1, pp. 136-147, 2003. 


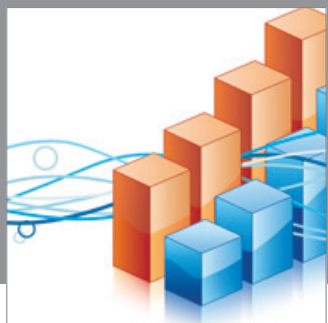

Advances in

Operations Research

mansans

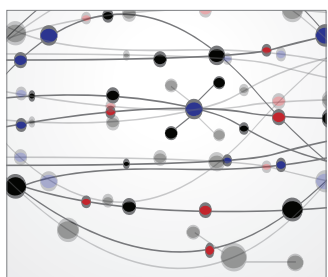

The Scientific World Journal
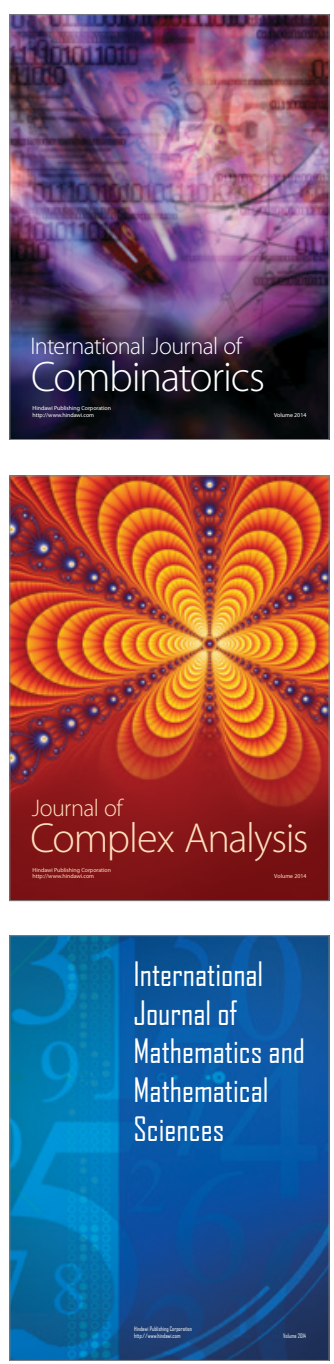
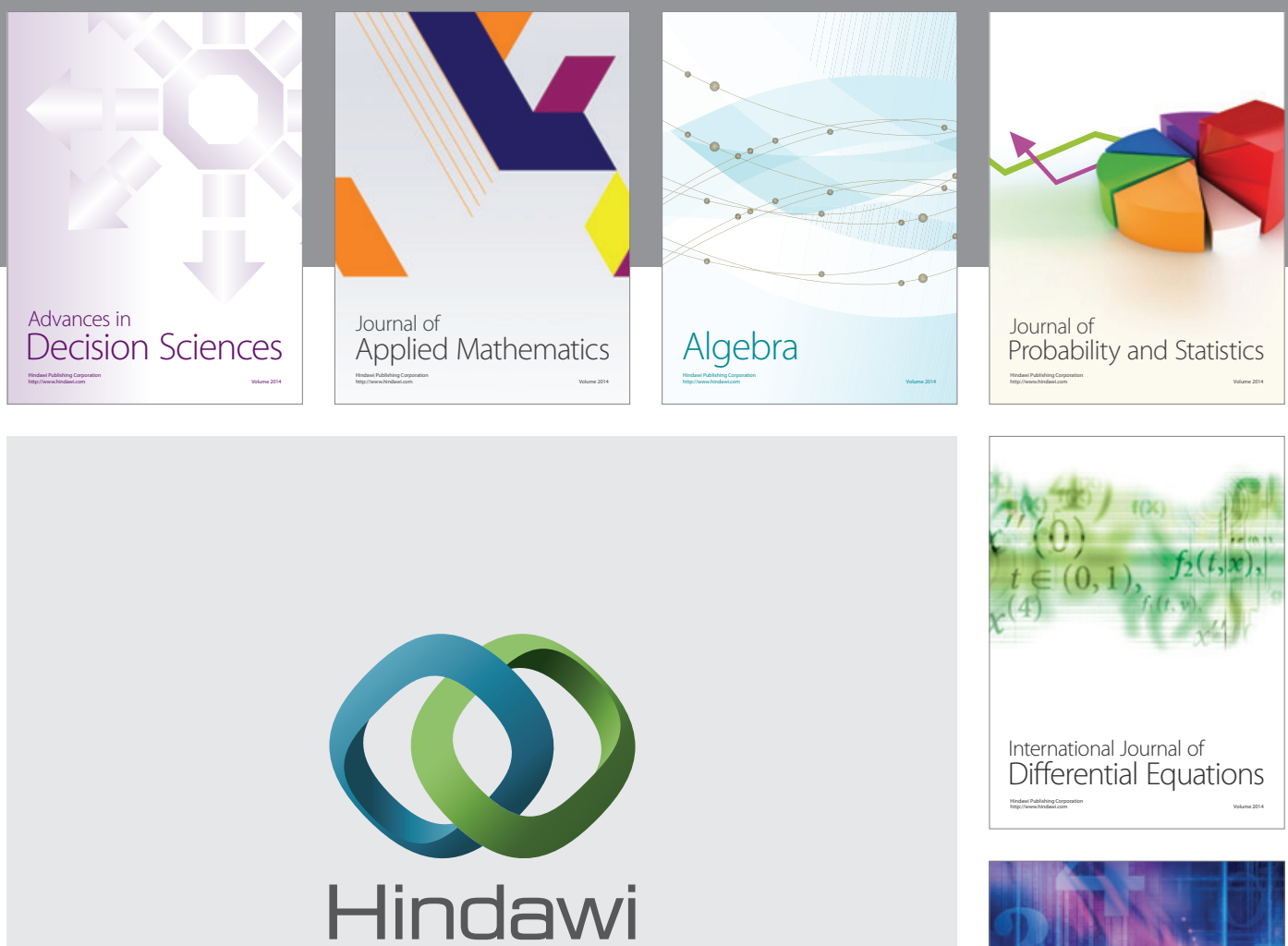

Submit your manuscripts at http://www.hindawi.com
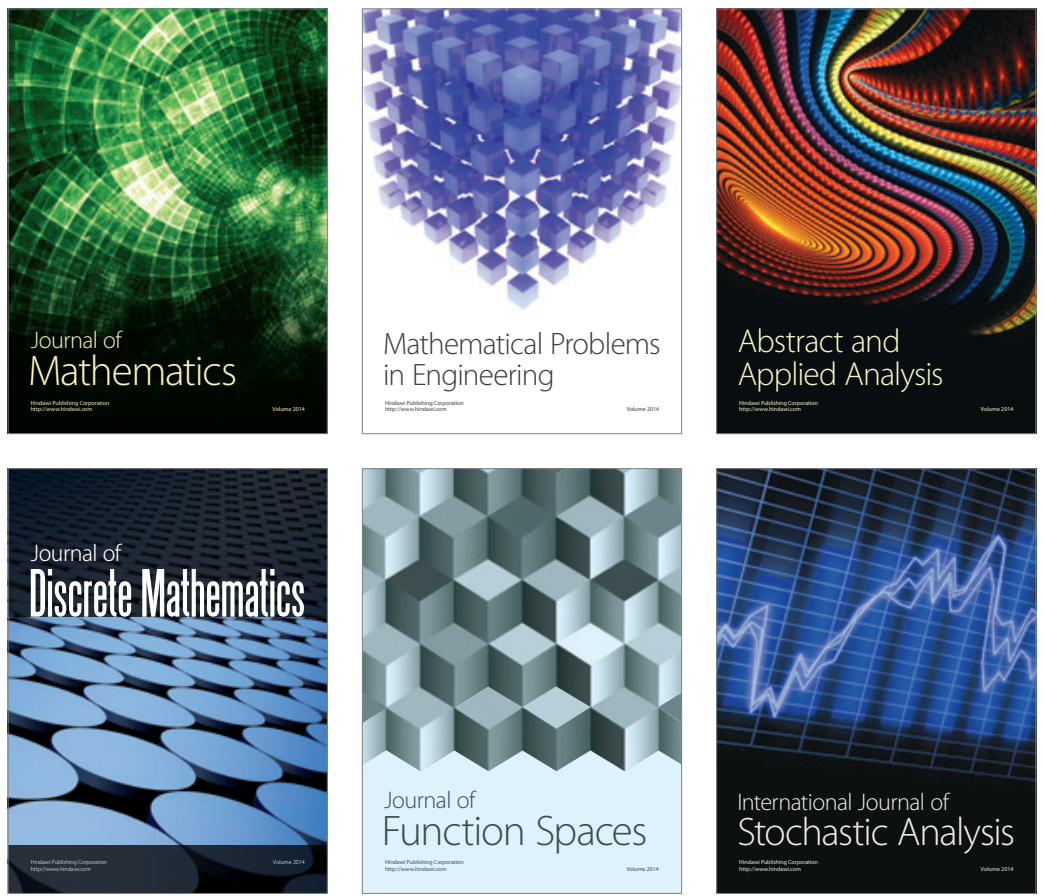

Journal of

Function Spaces

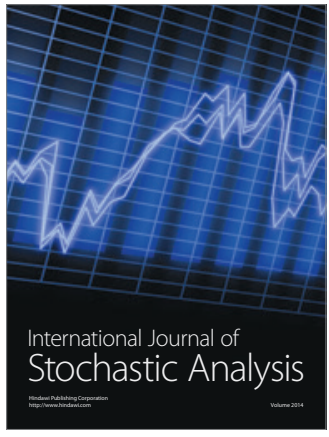

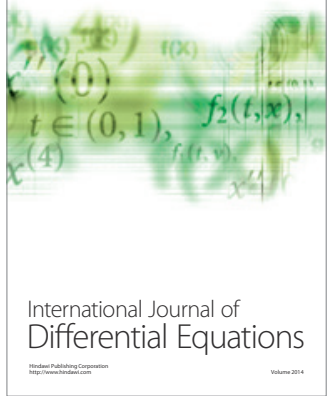
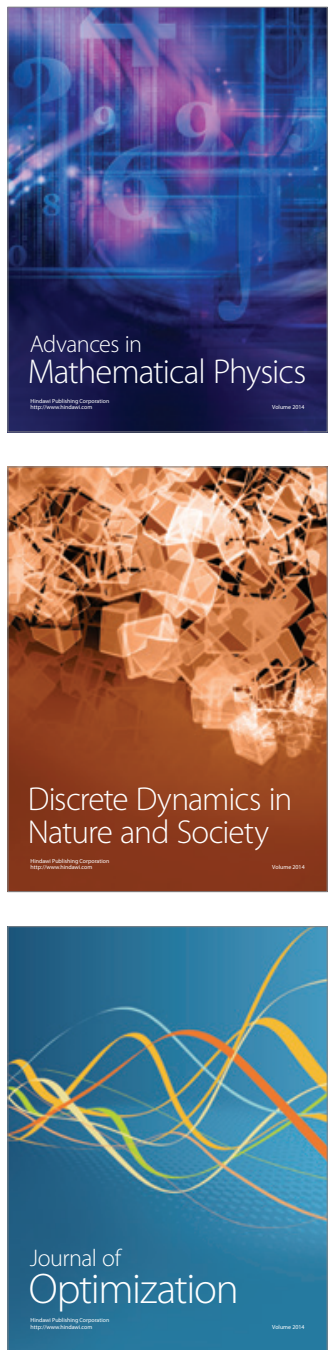